\title{
Le difficile suivi des déchets post-catastrophe : le cas de l'Ouragan Irma à Saint-Martin
}

\section{The difficult disaster waste management: case of Irma hurricane in Saint}

\section{Martin}

\author{
Hélène Beraud ${ }^{1}$, Charlotte Nithart ${ }^{2}$, Mathieu Durand ${ }^{3}$ \\ ${ }^{1}$ Lab’Urba, Université Paris-Est Marne-la-Vallée, helene.beraud@u-pem.fr \\ ${ }^{2}$ Robin des Bois, c.nithart@robindesbois.org \\ ${ }^{3}$ Le Mans Université, CNRS, UMR ESO, mathieu.durand@univ-lemans.fr
}

RÉSUMÉ. La gestion des déchets post-ouragan constitue un enjeu fondamental de la post-crise. Or, le suivi de ces nouveaux déchets n'est pas sans poser de difficultés. Une revue de la littérature portant sur le suivi des déchets postouragan sur plusieurs territoires caribéens et états-uniens et une enquête de terrain menée sur l'ile de Saint-Martin quelques mois après le passage de l'ouragan Irma rendent compte de difficultés communes dans le suivi des déchets post-ouragan (installation dans l'urgence des aires de stockage temporaire, difficultés à réintroduire certains flux de déchets dans leur filière de gestion habituelle, enfouissement massif, pollutions des eaux, du sol, dépôts sauvages de déchets...). Cet article met également en lumière quelques spécificités des milieux insulaires en matière de gestion des déchets (disponibilité du foncier, difficile accès à d'autres territoires, faible diversité des filières de traitement et de valorisation) qui rendent particulièrement difficile la gestion des déchets post-ouragan.

ABSTRACT. Hurricane waste management is a significant post-crisis issue. Based on review of articles on hurricane waste management in several Caribbean and US territories and on a survey a few months after Irma hurricane in the Saint Martin island, this article shows difficulties in the hurricane waste managament (inappropriate location of temporary debris staging; difficulties of sorting and recycling; water pollution; soil pollution; illegal dumping; open burning...). Also, this article highlights some specificities of waste management in island (difficult access to other territories; low diversity of treatment and recovery options). These specificities make particularly difficult to manage hurricane waste.

MOTS-CLÉS. Déchets post catastrophe, Ouragan, Caraïbes, Gisement, Gestion des déchets.

KEYWORDS. Disaster waste, Hurricane, Waste management, Island.

La gestion des déchets est un élément fondamental de la gestion de crise suite à des catastrophes naturelles. Juste après l'événement, les déchets gênent la progression des services de secours et la remise en état des réseaux techniques (eau, énergie...). Dans les jours qui suivent, le service de gestion des déchets doit faire face aux quantités exceptionnelles de déchets produites par l'événement s'ajoutant aux éventuels dysfonctionnements liés à la vulnérabilité des infrastructures. A plus long terme, une mauvaise gestion des déchets post-catastrophe peut engendrer des dommages environnementaux (pollutions), des risques sanitaires (moustiques et animaux nuisibles, brûlages à l'air libre), des dommages sociaux (rythme et choix de zones à déblayer), économiques (redémarrage de l'activité) et une dégradation de l'image des territoires (Beraud, 2013; Brown, 2012). A ces difficultés s'ajoutent des coûts de gestion extrêmement importants (à l'image des quantités produites), de l'ordre d'un tiers du coût total de redémarrage des territoires (FEMA, 2007). Les retours d'expériences et la littérature montrent une similitude des difficultés dans la gestion des déchets post catastrophe entre les territoires et les aléas. Ces difficultés semblent liées à plusieurs phénomènes : exutoires en nombre insuffisant, nécessité de ne pas saturer les capacités des filières de gestion existantes, impossibilité de se projeter en quantité et qualité dans la nature du gisement.

Les ouragans sont un aléa particulièrement générateur de déchets (Brown et al., 2011; FEMA, 2007; UNOCHA, 2011). En septembre 2017, l'ouragan Irma provoque des dégâts importants dans les îles des Caraïbes. Dans le cadre d'un projet de recherche ${ }^{1}$, s'intéressant à la gestion des déchets post-ouragan

${ }^{1}$ L'ANR DéPOs : Projet de recherche porté par le laboratoire Lab’Urba (Université Paris-Est-Marne-La-Vallée), l'IFSTTAR, le laboratoire ESO (UMR CNRS - Le Mans Université) et l'association Robins des bois visant à d'évaluer les quantités et types de déchets en fonction de la force et du 
dans les Antilles françaises, une enquête de terrain a été menée sur l'ile de Saint-Martin visant à décrire la manière dont les déchets post Irma ont été gérés (Robin des Bois, 2018). Les données recueillies montrent une gestion difficile. Il est alors important ici de bien comprendre les caractéristiques du gisement de déchets post-ouragan, ainsi que l'organisation des opérations de gestion. Les retours d'expériences portant sur d'autres territoires caribéens et états-uniens permettent de dégager quelques constantes d'organisation et de difficultés dans la gestion des déchets post-ouragan. Dans une deuxième partie, le suivi des déchets post-Irma à Saint-Martin est présenté. Cette mise en regard permet en conclusion de déterminer si les caractéristiques du gisement de déchets, les moyens de collecte et de traitement des déchets, les difficultés rencontrées sont similaires, et par là-même de questionner l'existence de spécificités du territoire dans la production de déchets post-ouragan et l'organisation des opérations de collecte et de traitement.

\section{Assurer le suivi des déchets post-ouragan : une obligation légale difficile à mettre en œuvre}

\begin{tabular}{|c|c|c|}
\hline Ouragan & $\begin{array}{l}\text { Territoires concernés dans les } \\
\text { publications étudiées }\end{array}$ & Références \\
\hline Hugo - 1989 & Etats-Unis, Guadeloupe & (EPA, 1995; Pagney Bénito-Espinal \& Bénito-Espinal, 1991) \\
\hline Iniki - 1992 & Hawaï (Etats-Unis) & (EPA, 1995; Reinhart \& McCreanor, 1999) \\
\hline Andrews - 1992 & Etats-Unis & $\begin{array}{l}\text { (EPA, 1995; FEMA, 2007; Reinhart \& McCreanor, 1999; Tansel } \\
\text { et al., 1994) }\end{array}$ \\
\hline Fran - 1996 & Etats-Unis & (FEMA, 2007) \\
\hline Georges - 1998 & Etats-Unis & (Reinhart \& McCreanor, 1999) \\
\hline Floyd - 1999 & Etats-Unis & (Escobedo et al., 2009) \\
\hline Frances et Jeanne - 2004 & Etats-Unis & $(\mathrm{EPA}, 2008)$ \\
\hline Ivan - 2004 & Etats-Unis, Grenade & $\begin{array}{l}\text { (Escobedo et al., 2009; UNDP Barbados \& OECS, 2007; World } \\
\text { Bank, 2004) }\end{array}$ \\
\hline Katrina - 2005 & Etats-Unis & $\begin{array}{l}\text { (Boxer \& Oberstar, 2008; Brandon et al., 2011; Brown \& Milke, } \\
\text { 2016; Brown et al., 2011; Dubey et al., 2007; EPA, 2018; } \\
\text { Hassett \& Handley, 2006; Luther, 2008; Moe, 2010; Roper, } \\
\text { 2008; SWANA, 2005) }\end{array}$ \\
\hline Dean - 2007 & Martinique & (Ravenet \& Vuillet) \\
\hline Ike - 2008 & Turques-et-Caïques & (UN Environment \& OCHA Joint Unit, 2008) \\
\hline Sandy - 2012 & Etats-Unis & $(\mathrm{EPA}, 2018)$ \\
\hline Harvey - 2017 & Etats-Unis & $(\mathrm{EPA}, 2018)$ \\
\hline Irma et Maria - 2017 & Dominique & (UN Environment \& OCHA Joint Unit, 2017) \\
\hline
\end{tabular}

Tableau 1. Liste des références étudiées

Depuis le $1^{\text {er }}$ janvier 2013, les Conseils régionaux ${ }^{2}$ ont l'obligation d'anticiper la gestion des déchets «de situations exceptionnelles » dans le cadre des plans régionaux de prévention et de gestion des déchets. En situation de crise, il faut en effet gérer les déchets de la catastrophe, tout en continuant à 
gérer les déchets produits au quotidien. L'objectif est ici de comprendre la manière dont le suivi des déchets post-ouragan a été mené sur différents territoires. Cette littérature recouvre essentiellement des catastrophes qui ont touchées les Etats-Unis, parmi lesquelles l'ouragan Katrina est particulièrement bien documenté. C'est en effet l'une des catastrophes naturelles qui a produit le plus grand volume de déchets.

\subsection{Les spécificités du gisement des déchets post-ouragan}

L'ensemble des retours d'expérience du tableau $\mathrm{n}^{\circ} 1$ montre que les déchets post-ouragan sont constitués par l'ensemble des biens, des stocks, des bâtiments, des infrastructures, de la végétation endommagé par un ouragan (Tableau 2). Ces dommages peuvent avoir plusieurs origines : l'action du $v^{v e n t}{ }^{3}$, les inondations et les glissements de terrain causés par les précipitations importantes, la marée cyclonique. La qualité et la quantité des déchets post-ouragan sont donc susceptibles de varier fortement en fonction des caractéristiques de chaque ouragan et des territoires impactés (Tableau 3). Par exemple, les déchets post-Katrina à la Nouvelle-Orléans sont issus pour partie de l'inondation provoquée par la rupture des digues du Lac Pontchartrain. L'inondation des habitations sous plusieurs mètres d'eau pendant plusieurs semaines a généré des déchets dont la nature et les caractéristiques se rapprochent de celles des déchets post-inondation : matériaux de construction gorgées d'eau, affaires personnelles, $\mathrm{DEEE}^{4}$ en quantités très importantes. Ainsi, la nature des déchets dans cette ville n'est pas tout à fait la même que celle observée dans les autres comtés touchés par Katrina (Luther, 2008).

\begin{tabular}{|c|c|c|c|c|c|c|c|c|}
\hline $\begin{array}{l}\text { Type de déchets } \\
\text { Type d'aléa }\end{array}$ & 足 & 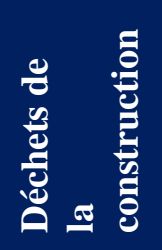 & 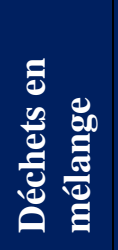 & 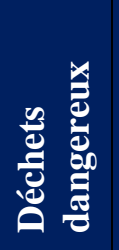 & $\frac{\frac{\sqrt{x}}{x-2}}{\frac{x}{2}}$ & 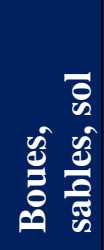 & 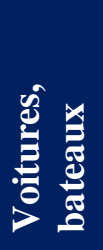 & 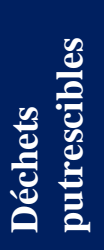 \\
\hline $\begin{array}{l}\text { Ouragan / cyclone / } \\
\text { typhon }\end{array}$ & $\mathrm{X}$ & $\mathrm{X}$ & $X$ & $\mathrm{X}$ & $\mathrm{X}$ & $\mathrm{X}$ & $\mathrm{X}$ & $\mathrm{X}$ \\
\hline Tsunamis & $\mathrm{X}$ & $X$ & $\mathrm{X}$ & $X$ & $\mathrm{X}$ & $X$ & $\mathrm{X}$ & $X$ \\
\hline Tornade & $\mathrm{X}$ & $\mathrm{X}$ & $\mathrm{X}$ & $\mathrm{X}$ & $\mathrm{X}$ & & $\mathrm{X}$ & $\mathrm{X}$ \\
\hline Inondation & $\mathrm{X}$ & $X$ & $X$ & $X$ & $X$ & $\mathrm{X}$ & $\mathrm{X}$ & $\mathrm{X}$ \\
\hline Tremblement de terre & & $\mathrm{X}$ & $X$ & $X$ & $\mathrm{X}$ & $\mathrm{X}$ & & \\
\hline
\end{tabular}

Tableau 2. Type de déchets produits en fonction de la nature de l'aléa (adapté de (FEMA, 2007)

Le tableau $\mathrm{n}^{\circ} 3$ recense pour quelques ouragans des informations sur la quantité des déchets produits variant de quelques centaines de milliers de tonnes à plusieurs dizaines de millions de tonnes. A quelques rares exceptions, comme à la Nouvelle-Orléans par exemple, ces quantités de déchets ont été produites en quelques heures seulement. Cette production exceptionnelle rapportée en année de production de déchets de routine (10 ans de production de déchets verts pour Hugo, 7 ans de production pour Iniki, 6 ans de production pour Katrina dans le Mississippi) rend compte des conséquences potentielles sur le fonctionnement du service de gestion des déchets.

\footnotetext{
${ }^{3}$ Celle-ci peut être renforcée par la formation de tornades. Les ouragans créent en effet les conditions favorables à la formation de ce type de phénomènes (FEMA, 2007).

${ }^{4}$ DEEE : déchets d'équipements électroniques et électriques.
} 


\begin{tabular}{|c|c|c|c|c|c|c|}
\hline Ouragan & $\begin{array}{l}\text { Quantité de } \\
\text { déchets }\end{array}$ & $\begin{array}{c}\text { Répartition par } \\
\text { nature de } \\
\text { déchets }\end{array}$ & $\begin{array}{l}\text { Eléments sur } \\
\text { les dommages }\end{array}$ & $\begin{array}{l}\text { Conversion } \\
\text { en année de } \\
\text { production }\end{array}$ & $\begin{array}{c}\text { Principaux } \\
\text { flux de déchets } \\
\text { post ouragan } \\
\text { triés }\end{array}$ & Sources \\
\hline $\begin{array}{l}\text { Hugo } \\
\text { (Caroline du } \\
\text { Nord, Etats- } \\
\text { Unis, 1989) }\end{array}$ & & $\begin{array}{l}2 \text { millions cy }{ }^{5} \text { de } \\
\text { déchets verts }\end{array}$ & & $\begin{array}{l}10 \text { années } \\
\text { pour déchets } \\
\text { verts }\end{array}$ & Déchets verts & $\begin{array}{l}\text { (EPA, 1995; } \\
\text { Reinhart \& } \\
\text { McCreanor, } \\
1999 \text { ) }\end{array}$ \\
\hline $\begin{array}{l}\text { Iniki } \\
\text { (Hawaï, 1992) }\end{array}$ & 5 millions cy & & & 7 années & $\begin{array}{l}\text { Déchets verts, } \\
\text { Métaux, Bois } \\
\text { (construction), } \\
\text { Déchets de la } \\
\text { construction } \\
\text { (plusieurs sous } \\
\text { flux : } \\
\text { panneaux de } \\
\text { gypse, } \\
\text { toiture,...), } \\
\text { Déchets } \\
\text { dangereux }\end{array}$ & $\begin{array}{l}\text { (Reinhart \& } \\
\text { McCreanor, } \\
\text { 1999) }\end{array}$ \\
\hline $\begin{array}{l}\text { Andrews } \\
\text { (Floride, Etats- } \\
\text { Unis, 1992) } \\
\text { Comté Metro- } \\
\text { Dade (Grand } \\
\text { Miami) }\end{array}$ & $\begin{array}{l}43 \text { millions cy } \\
6 \text { millions de } \\
\text { tonnes }\end{array}$ & $\begin{array}{l}50 \% \text { de déchets } \\
\text { de la } \\
\text { construction }\end{array}$ & $\begin{array}{l}150000 \\
\text { maisons } \\
\text { endommagées } \\
\text { / détruites }\end{array}$ & & $\begin{array}{l}\text { Déchets verts, } \\
\text { Métaux, } \\
\text { ferrailles, } \\
\text { Déchets } \\
\text { d'équipements } \\
\text { électroniques } \\
\text { et électriques }\end{array}$ & $\begin{array}{l}\text { (Reinhart \& } \\
\text { McCreanor, } \\
\text { 1999) } \\
(\text { EPA, 1995) }\end{array}$ \\
\hline $\begin{array}{l}\text { Floyd } \\
\text { (Caroline du } \\
\text { Nord, Etats- } \\
\text { Unis, 1999) }\end{array}$ & & $\begin{array}{l}70 \% \text { de déchets } \\
\text { verts }\end{array}$ & & & & $\begin{array}{l}\text { (Escobedo et } \\
\text { al., 2009) }\end{array}$ \\
\hline $\begin{array}{l}\text { Frances et } \\
\text { Jeanne (Comté } \\
\text { de Palm } \\
\text { Beach, } \\
\text { Floride, Etats- } \\
\text { Unis, 2004) } \\
\end{array}$ & 6 millions cy & $\begin{array}{l}80 \% \text { de déchets } \\
\text { verts }\end{array}$ & & & Déchets verts & (EPA, 2008) \\
\hline $\begin{array}{l}\text { Ivan (Grenade, } \\
\text { 2004) }\end{array}$ & & $\begin{array}{l}50 \% \text { des arbres } \\
\text { de la Réserve de } \\
\text { Grand Etang } \\
\text { Forest. }\end{array}$ & $\begin{array}{l}100 \% \text { de la } \\
\text { culture banane } \\
\text { détruite } \\
40 \% \text { des } \\
\text { maisons } \\
\text { inhabitables }\end{array}$ & & & $\begin{array}{l}\text { (World Bank, } \\
\text { 2004) }\end{array}$ \\
\hline $\begin{array}{l}\text { Ivan (Comté } \\
\text { d'Escambia, } \\
\text { Floride, Etats- } \\
\text { Unis, 2004) }\end{array}$ & 10 millions cy & $\begin{array}{l}6,5 \text { millions cy } \\
\text { de déchets verts } \\
1,35 \text { million cy } \\
\text { de sables } \\
\text { déplacés par } \\
\text { l'ouragan }\end{array}$ & & & & $\begin{array}{l}\text { (EPA, 2008; } \\
\text { Escobedo et al., } \\
2009)\end{array}$ \\
\hline $\begin{array}{l}\text { Katrina } \\
\text { (Louisiane, } \\
\text { Mississippi, } \\
\text { Etats-Unis, } \\
\text { 2005) }\end{array}$ & $\begin{array}{l}100 \text { millions cy } \\
\left(76 \text { millions } \mathrm{m}^{3}\right) \\
\text { dont } 3 \text { millions } \\
\text { cy pour } \\
\text { l'Alabama, } 45 \\
\text { millions cy pour }\end{array}$ & $\begin{array}{l}50 \% \text { déchets de } \\
\text { la construction } \\
30 \% \text { de déchets } \\
\text { verts } \\
13 \text { millions de }\end{array}$ & $\begin{array}{l}600000 \\
\text { maisons } \\
\text { impactées dont } \\
77 \% \\
\text { totalement } \\
\text { détruites }\end{array}$ & $\begin{array}{l}\text { Mississippi : } \\
6 \text { années de } \\
\text { production } \\
\text { normale de } \\
\text { l'Etat }\end{array}$ & $\begin{array}{l}\text { Déchets verts, } \\
\text { Déchets de la } \\
\text { construction, } \\
\text { Déchets } \\
\text { d'équipements }\end{array}$ & $\begin{array}{l}\text { (Boxer \& } \\
\text { Oberstar, 2008; } \\
\text { Dubey et al., } \\
\text { 2007; EPA, } \\
\text { 2018; Hassett \& } \\
\text { Handley, 2006; }\end{array}$ \\
\hline
\end{tabular}

${ }^{5} \mathrm{cy}=$ cubic yard. Le facteur de conversion est le suivant : $1 \mathrm{cy}=0,764555 \mathrm{~m}^{3}$ 


\begin{tabular}{|c|c|c|c|c|c|c|}
\hline & $\begin{array}{l}\text { le Mississippi et } \\
64 \text { millions cy } \\
\text { pour la } \\
\text { Louisiane } \\
22 \text { millions de } \\
\text { tonnes pour le } \\
\text { sud de la } \\
\text { Louisiane } \\
\text { (Nouvelle- } \\
\text { Orléans } \\
\text { notamment) }\end{array}$ & $\begin{array}{l}\mathrm{m}^{3} \text { de déchets de } \\
\text { bois de } \\
\text { construction } \\
\text { En Louisiane : } \\
\text { - environ 1,5 } \\
\text { millions d'unités } \\
\text { d'équipements } \\
\text { électriques et } \\
\text { électroniques } \\
\text { - environ } 7 \\
\text { millions de } \\
\text { tonnes de } \\
\text { déchets } \\
\text { dangereux } \\
\text { - } 16 \text { millions de } \\
\text { tonnes de } \\
\text { déchets } \\
\text { putrescibles } \\
\text { - } 350000 \\
\text { voitures } \\
\text { - } 60000 \text { bateaux }\end{array}$ & $\begin{array}{l}\text { (Louisiane) } \\
63500 \\
\text { maisons } \\
\text { inhabitables } \\
\text { (Mississippi) }\end{array}$ & $\begin{array}{l}\text { Nouvelle- } \\
\text { Orléans : } \\
\text { production } \\
\text { moyenne } \\
\text { annuelle } 350 \\
000 \text { tonnes. }\end{array}$ & $\begin{array}{l}\text { électroniques } \\
\text { et électriques, } \\
\text { Véhicules, } \\
\text { Bateaux, } \\
\text { Déchets } \\
\text { dangereux }\end{array}$ & $\begin{array}{l}\text { Luther, 2008; } \\
\text { Moe, 2010; } \\
\text { Roper, 2008) }\end{array}$ \\
\hline Ike (2008) & & & & & $\begin{array}{l}\text { Déchets } \\
\text { métalliques et } \\
\text { épaves }\end{array}$ & $\begin{array}{l}\text { (UN } \\
\text { Environment } \\
\text { \& OCHA } \\
\text { Joint Unit, } \\
\text { 2008) }\end{array}$ \\
\hline $\begin{array}{l}\text { Sandy (New- } \\
\text { York, Etats- } \\
\text { Unis, 2012) }\end{array}$ & 700000 tonnes & & & & $\begin{array}{l}\text { Déchets } \\
\text { métalliques et } \\
\text { épaves }\end{array}$ & (EPA, 2018) \\
\hline $\begin{array}{l}\text { Harvey } \\
\text { (Texas, Etats- } \\
\text { Unis, 2017) }\end{array}$ & $\begin{array}{l}11,8 \text { millions cy } \\
\text { de déchets } \\
\text { collectés en } \\
\text { février } 2018 \\
\text { (estimation } \\
\text { FEMA : } \\
\text { représente } 75 \% \\
\text { du gisement } \\
\text { total) }\end{array}$ & & & & & (EPA, 2018) \\
\hline
\end{tabular}

Tableau 3. Quantité de déchets produits par quelques ouragans

Les déchets verts et les déchets de la construction représentent la part la plus importante des déchets produits. A la différence des seconds, les déchets verts (arbres déracinés, couchés, branches arrachées,...) sont particulièrement spécifiques des déchets post-ouragan (Escobedo et al., 2009; Pagney Bénito-Espinal \& Bénito-Espinal, 1991; Ravenet \& Vuillet). Une étude menée par Staudhammer et al. en 2009, citée par (Thompson et al., 2011), montre que, durant la saison des ouragans 2004-2005, les paroisses ${ }^{6}$ de Floride avaient dépensé entre 3000 dollars et 4 millions de dollars par tempête pour la gestion de ces déchets, avec une moyenne de 704045 dollars par commune et par tempête ${ }^{7}$. Outre sa vulnérabilité aux ouragans, le couvert végétal est particulièrement étudié car il est considéré comme un facteur de réduction des impacts des ouragans en milieu urbain (Van der Sommen et al., 2018). Les déchets de la construction, moins spécifique aux ouragans ${ }^{8}$, représentent

\footnotetext{
${ }^{6}$ Nous avons traduit le terme « community $»$ par « paroisse ».

7 « During the 2004-2005 hurricane seasons, communities in the state of Florida in the United States (US) spent between US \$3000 and $\$ 4$ million per storm on clean-up and disposal of vegetation debris, with an average of US \$704,045 per community, per storm (Staudhammer et al., 2009) » p. 286 (Thompson et al., 2011)

${ }^{8}$ Les tremblements de terre produisent essentiellement ce type de déchets, et en quantité très importante.
} 
également une part importante des déchets produits. Ce gisement pose des difficultés de gestion car son recyclage ou sa réutilisation ne sont pas aisés, notamment en raison du mélange des matériaux. Néanmoins, les nombreuses réflexions sur les constructions anti-cycloniques ont vocation à contribuer à sa réduction (Aquino et al., 2018; Tansel et al., 1994).

\subsection{Les opérations de gestion des déchets post-ouragan}

La gestion des déchets post-ouragan recouvre les mêmes étapes que la gestion des déchets de routine : la collecte, le tri, le traitement (réutilisaton, recyclage ou élimination). Il est à noter que cette gestion se superpose systématiquement à la reprise (ou continuité) des opérations de gestion des déchets de routine.

\subsubsection{Des opérations de gestion entre urgence et nécessité de maintien sur le temps long}

La littérature découpe généralement la gestion des déchets post-catastrophe en trois périodes. Une première phase d'urgence où l'objectif est de déblayer rapidement les routes afin de permettre l'accès aux secours et la remise en état des infrastructures vitales, de collecter les déchets susceptibles de présenter des risques sanitaires et environnementaux. Une seconde phase de nettoyage durant laquelle la majorité des déchets sont collectés, traités, et les opérations de déconstruction commencent. Une troisième phase, beaucoup plus longue, qui est celle de la déconstruction/reconstruction (générant des déchets de la construction) et de la remise en état des installations ayant permis la gestion des déchets post-catastrophe (Beraud, 2013; Brown et al., 2011; Roper, 2008). Cette dernière phase est particulièrement longue et incertaine. Elle peut durer plusieurs mois, voire plusieurs années (Boxer \& Oberstar, 2008; EPA, 1995; Luther, 2008). Par exemple, le nettoyage des déchets après le passage de l'ouragan Andrews, en 1992, dans le comté de l'agglomération de Miami (Miami-Dade) a duré 4 ans (Reinhart \& McCreanor, 1999). Pour la gestion des déchets dans l'Etat du Mississippi suite au passage de Katrina, en 2005, les experts estimaient en 2006 qu'il faudrait cinq ans pour gérer l'ensemble des déchets post-ouragan (Hassett \& Handley, 2006).

Ce phasage se matérialise sur le terrain par des variations fortes des déchets à collecter et à traiter, nécessitant une adaptation des capacités de collecte et, in fine, des capacités de traitement. Par exemple, les Nations-Unies (UN Environment \& OCHA Joint Unit, 2008) montrent que sur l'ile de Grand Turk, suite au passage de l'ouragan Ike en septembre 2008, les volumes qui arrivaient quotidiennement à la décharge, en octobre, étaient 10 à 15 fois supérieurs à ceux qui arrivent en période normale. Ensuite, en décembre, le volume de déchets ne représentait plus que l'équivalent de 2 fois le volume produit en période normale. Les zones de stockage temporaire, que nous allons décrire par la suite, permettent d'atténuer quelques peu cette pression sur les exutoires.

\subsubsection{De la collecte au tri : la place centrale du stockage temporaire}

La phase de collecte des déchets est généralement réalisée en porte à porte. Dans certains cas, les autorités ont diffusé des messages invitant par exemple les sinistrés à bien différencier les déchets de routine des déchets post-ouragan (EPA, 2008).

Les déchets sont ensuite le plus souvent acheminés vers des aires de stockage temporaire. Ces aires offrent la possibilité aux autorités de bénéficier d'un espace de régulation des flux permettant de ne pas encombrer les exutoires. Dans la plupart des cas, ces aires ont été définies au cours de la post crise. Ainsi, après l'ouragan Andrews en Floride, ce n'est que suite à la saturation des déchetteries et des sites de transit préexistants, que le comté de Metro-Dade a ouvert des aires de stockage temporaire. En Caroline du Nord, 11 aires de stockage temporaires ont dû être ouvertes dans les jours qui suivent le passage de l'ouragan Hugo (EPA, 1995). Dans certains cas, les zones de stockage pré-identifiées n'ont pas été suffisantes; d'autres ont dû être trouvées dans l'urgence (Comté d'Escambia, Ivan, 2004, (EPA, 2008). 
Un tri des déchets est parfois effectué. Dans certains cas, il est pratiqué par les habitants devant leur habitation et/ou après collecte, sur les zones de stockage temporaires, les sites de transit ou sur les déchetteries déjà existantes. Il existe une diversité de situations suivant les territoires et les ouragans, en fonction des flux triés, du moment où se déroule cette étape. Une constante est observable dans le cas des ouragans, c'est le tri quasi systématique des déchets verts (Error! Reference source not found.). Cela s'explique par leur quantité importante, la facilité de leur tri (ils sont peu mélangés, facilement séparables des autres gisements).

Katrina et Iniki se distinguent des autres ouragans par un tri de plusieurs flux de déchets. Ces deux cas sont particuliers : Katrina a généré des déchets en quantité très importante, obligeant les autorités à se positionner pour le recyclage, après quelques semaines d'enfouissement massif, de réouverture des décharges fermées et de brûlage à l'air libre, engendrant des problèmes environnementaux et des plaintes de la population (Boxer \& Oberstar, 2008; Luther, 2008; Roper, 2008). Iniki, nous y reviendrons dans les paragraphes suivants, a touché un territoire insulaire sur lequel les solutions de gestion des déchets de routine étaient très limitées (EPA, 1995). A ces deux exceptions près, les démarches de tri et de recyclage sont peu développées. Mettre en place un tri spécifique nécessite de pouvoir facilement séparer les déchets, d'avoir les moyens techniques, humains, les surfaces disponibles, mais aussi et surtout les filières de traitement. Dans le cas d'Iniki et de l'ile de Kauai Hawaï, les autorités ont défini les flux de déchets à trier en fonction de l'existance de filières de gestion appropriées (EPA, 1995).

Charlotte Brown et Mark Milke, dans un article publié en 2016, sur les conditions de développement du recyclage des déchets post catastrophe, montrent la faible part des déchets post Katrina recyclés et tentent d'en expliquer les raisons. Ainsi, les auteurs citent entre autres des contraintes de temps imposés par les prestataires, la durée du recyclage (plus lent que l'enfouissement), le mélange des déchets et leur pollution (Brown \& Milke, 2016). Ils mettent également en évidence le fait que les capacités d'enfouissement ${ }^{9}$ étant importante en Louisiane, les coûts sont restés faibles. Seuls les problèmes de pollution ont conduit à la mise en œuvre d'autres solutions (cf. § suivants).

\subsubsection{Le traitement : des déchets en grande partie enfouis}

Une fois les déchets collectés, parfois triés, puis regroupés, plusieurs schémas de traitement sont possibles : soit le déchet est valorisé sur place (exemple des déchets verts qui, dans de nombreux cas, ont été déchiquetés sur les aires de stockage temporaire), soit orienté vers des filières de traitement spécifique (recyclage ou dépollution), soit éliminé (enfouissement ou incinération). (Brandon et al., 2011), dans un article sur le recyclage en Louisiane des déchets post-Katrina, montre ainsi la diversité des types de traitement et des exutoires pour différents flux de déchets.

Plus généralement, la littérature met en évidence que les déchets bénéficiant le plus souvent d'un recyclage sont les déchets verts ${ }^{10}$. Ces déchets sont généralement déchiquetés et utilisés ensuite comme combustibles de chauffage, paillis ou compost (après maturation). Cependant, les volumes produits étant tellement importants, certains exutoires ont rapidement été saturés. Ainsi, en Floride, dans le Comté d'Escambia, suite au passage de l'ouragan Ivan, le comté a dû exporter $60 \%$ des 6,5 millions de cy de déchets verts vers l'Italie (EPA, 2008). Les sables et boues sont également généralement bien réutilisés (EPA, 2008, EPA, 2018). EPA (2018) montre les économies réalisées par la ville de NewYork suite au choix de réutiliser les 2 millions cy de sable collecté.

Quelques expériences de recyclage de métaux, de DEEE, de véhicules, d'épaves, de déchets dangereux sont également observables. Par exemple en Louisiane, 7300 tonnes de déchets dangereux des ménages ont été collectés. Une partie importante a été recyclée notamment les piles, les bouteilles

\footnotetext{
${ }^{9}$ La littérature reprend souvent le terme anciennement utilisé «d'élimination » en tant que synonyme «d'enfouissement ». Il est maintenant acté que l'enfouissement n'est que du stockage de déchets, en rien de l'élimination.

${ }^{10}$ Cela n'empêche pas d'observer de nombreux cas de brûlage de déchets verts (Brandon et al., 2011)
} 
de propane, d'essence et de pétrole (EPA, 2008; Luther, 2008). Plus de 600000 équipements électriques et électroniques endommagés ont été collectés et recyclés en Louisiane. Enfin, bien que produits en quantité importante également, les déchets de la construction sont officiellement généralement peu valorisés. Seuls quelques exemples de réutilisation de matériaux de construction sont décrites dans la littérature (Brown \& Milke, 2016; Reinhart \& McCreanor, 1999; Tansel et al., 1994).

Finalement, une fois les déchets verts retirés, une part importante du volume de déchets produits est éliminée par enfouissement, incinération ou brûlage ${ }^{11}$. En Floride, après l'ouragan Andrews, le brûlage des déchets a été autorisé. Il a été préféré à l'enfouissement suite aux difficultés rencontrées par la Caroline du Nord trois ans auparavant pour gérer les déchets post-Hugo. Ces déchets avaient « consommés » l'équivalent de 14 années de capacité d'enfouissement de déchets de routine (Reinhart $\&$ McCreanor, 1999). Dans la majorité des cas, les déchets post-ouragan ont été enfouis soit dans des installations existantes, soit dans de nouvelles installations de stockage. Dans l'Etat du Mississippi, rapidement après Katrina, le Mississippi Department of Environmental Quality (MDEQ) s'est aperçu que les capacités des installations existantes d'enfouissement des déchets de la construction ne seraient pas suffisantes pour absorber le gisement de déchets produits. Le MDEQ souhaitait par ailleurs ne pas utiliser toutes les capacités des installations de stockage existantes. Face au mélange des déchets rendant leur valorisation difficile, il a donc décidé d'ouvrir 13 unités temporaires de stockage des déchets de construction. Cette ouverture d'unités de stockage dans l'urgence a nécessité quelques délais car les autorités ont pris des précautions pour leur localisation (vérification de la qualité des sols, de l'absence de nappes souterraines,...) (EPA, 2018).

\subsection{Les déterminants du pilotage du suivi des déchets post-ouragan}

\subsubsection{Préexistence d'une planification de la gestion des déchets post-ouragan}

Pour faire face aux difficultés potentielles liées au gisement de déchets post-ouragan et aux évolutions des moyens humains et techniques nécessaires à leur gestion, la littérature montre le rôle positif joué par la préexistence d'une planification. Un tel document, lorsqu'il anticipe les moyens nécessaires à la gestion des déchets post catastrophe, la localisation et la préparation des zones de stockage temporaire, limite les difficultés rencontrées en post crise $^{12}$. Par exemple, Julia Crowley montre, dans une étude réalisée sur 95 comtés des Etats-Unis, ayant connu une catastrophe majeure entre 2012 et 2015, que ceux dotés d'une planification de la gestion des déchets post catastrophe avant l'événement ont recyclé deux fois plus de déchets (Crowley, 2017). Le comté de Palm Beach a par exemple mis en place un Plan de gestion des déchets post catastrophe après l'Ouragan Irene de 1999. Ce plan contient des informations sur la localisation et la sécurisation des infrastructures de stockage temporaire, sur le nettoyage des routes, sur les entreprises prestataires à mobiliser pour gérer les déchets post-ouragan (EPA, 2008). L'anticipation d'une telle organisation a permis aux autorités de communiquer, avant même le passage des ouragans Frances et Jeanne en 2004, sur les consignes de tri, et de positionner rapidement des moyens d'actions après leur passage. Si l'existence d'un plan est une réelle plus-value, elle n'est pas suffisante (Crowley, 2017). Le comté d'Escambia avait par exemple un plan de gestion. Toutefois, n'ayant pas identifié les entreprises prestataires, il lui a fallu 12 jours pour

\footnotetext{
${ }^{11}$ L'incinération est ici différenciée du brûlage des déchets par, dans le premier cas, l'utilisation d'une infrastructures d'incinération des déchets normaux. Dans le second cas, cette technique d'élimination est un dispositif d'urgence, mis en place pour gérer le surplus de déchets.

${ }^{12}$ La formalisation d'un tel plan nécessite cependant de connaitre la nature et l'ampleur du gisement post catastrophe pour pouvoir anticiper les dysfonctionnements potentiels du service et pouvoir dimensionner les moyens nécessaires. Cette connaissance n'est pas aisée à obtenir. Elle nécessite l'usage d'une méthode d'estimation des déchets avant la catastrophe s'appuyant sur les caractéristiques du territoire. Si quelques unes permettent un tel calcul de manière globale, pour un type de déchets (Escobedo et al., 2009; Tansel et al., 1994; Thompson et al., 2011)), ou pour quelques principaux flux (COES, 2005; FEMA, 2006; Umpierre \& Margoles, 2011), peu proposent une quantification en fonction de différents flux de déchets (ici pour les déchets issus des inondations (Beraud et al., 2012). Or, c'est cette dernière solution qui permet de réintroduire un maximum de déchets dans une filière de gestion spécifique. Cela explique partiellement pourquoi beaucoup de territoire rencontrent encore des difficultés à anticiper la gestion des déchets postcatastrophe. Le projet ANR DéPOs propose de travailler à formalisation d'une telle méthode de quantification pour les déchets postouragan.
} 
les sélectionner et rédiger les contrats (EPA, 2008). Dans la majorité des cas étudiés, la question des déchets avait été négligée dans les plans de préparation à la gestion de crise.

\subsubsection{Influence des infrastructures existantes sur le choix des modes de gestion}

Outre la réaction rapide permise par l'existence de plans, plus globalement, il s'agit de réfléchir dès l'amont aux solutions de traitement envisageables. La littérature montre que l'état des capacités de gestion des déchets normaux a été un facteur déterminant lors de la définition de la stratégie de gestion des déchets post catastrophe. Quand l'ouragan Hugo s'est abattu sur les côtes de Caroline du Nord, la décharge du comté de Macklenburg n'avait plus que l'équivalent de 2,5 années de capacité de stockage. Cette situation a imposé un choix au final plus vertueux de déchiqueter les déchets verts produits pour les utiliser en combustible de chauffage ou en paillis (EPA, 2008). Sur l'ile de Kauai Hawaï en 1992, suite au passage de l'ouragan Iniki, les autorités ont dû faire face à une production de déchets équivalente à sept années de routine, alors que leur décharge avait une capacité de traitement de moins de quatre ans, sans aucune capacité de transport des déchets vers une autre île. Les autorités ont alors mis en place un recyclage d'un maximum de flux (EPA, 1995), nécessitant alors une réflexion, dans l'urgence, qui toutefois a duré trois mois.

A l'inverse, certains territoires sont moins contraints par la disponibilité des installations de traitement. La Louisiane disposait par exemple, au moment du passage de Katrina, d'une capacité de stockage des déchets suffisamment importante pour ne pas envisager, dans un premier temps, leur tri (EPA, 2018). Ces fortes capacités d'enfouissement pour les déchets de construction ont amené les autorités à autoriser un enfouissement massif sans même un tri préalable des déchets dangereux. Or, les centres d'enfouissement n'étaient pas habilités au stockage de ce type de déchets, engendrant une pollution incitant les autorités à revoir leur stratégie (Boxer \& Oberstar, 2008; EPA, 2018; Roper, 2008).

\subsubsection{De la nécessaire coordination des moyens : une gestion multi-acteurs}

Comme nous le montre les références étudiées, le gisement de déchets post-ouragan impose aux gestionnaires de déchets d'une part, aux gestionnaires de la crise d'autre part, d'organiser une gestion des déchets qui ne peut se satisfaire des moyens dévolus à la gestion des déchets de routine. Cette observation amène à s'interroger sur le positionnement du service de gestion des déchets de routine dans la gestion de l'exceptionnel. Peu de travaux analysent la manière dont le service de gestion des déchets s'est réorganisé en période de post crise face à la production importante de déchets ou face aux dysfonctionnements du service de gestion normale (Durand et al., 2015; Popescu et al., 2014).

Si l'on se concentre uniquement sur l'organisation de la gestion des déchets post-ouragan, la situation d'urgence et le gisement important de déchets nécessite la mobilisation de nombreux acteurs. Par exemple, aux Etats-Unis, outre les autorités en charge de la définition de la stratégie, plusieurs agences fédérales jouent un rôle important $\left(\mathrm{FEMA}^{13}, \mathrm{EPA}^{14}, \mathrm{USACE}^{15}\right)($ Boxer \& Oberstar, 2008; Luther, 2008; Moe, 2010). Lorsque l'Etat de Louisiane a pris la décision de diversifier les solutions de traitement des déchets post-Katrina et de développer le recyclage, il s'est appuyé sur l'EPA et l'USACE. L'EPA a accompagné le LDEQ (Louisiane Départment of Environmental Quality) dans la rédaction du plan de collecte et de recyclage. Avec l'USACE, elles ont également vérifié la bonne application des consignes de recyclage en pilotant la collecte et le tri des déchets dans plusieurs paroisses. La FEMA est intervenue dans le financement de la gestion de crise, et notamment dans le remboursement des Comtés et des Etats. Cette mobilisation d'acteurs en dehors du champ de la gestion des déchets de routine s'observe sur les autres cas d'études. La gestion des déchets post catastrophe est partie intégrante des opérations de gestion de la post-catastrophe.

\footnotetext{
${ }^{13}$ Federal Emergency Management Agency

${ }^{14}$ Environmental Protection Agency

${ }^{15}$ US Army Corps of Engineers
} 
Outre, les agences fédérales et les administrations, des entreprises sont également mobilisées pour participer à certaines étapes de la gestion des déchets. L'intervention des entreprises est le plus souvent formalisée par la signature de contrats. La littérature insiste sur la durée de rédaction et de signature de ces contrats qui peuvent retarder les opérations de collecte, ainsi que sur la nécessité de définir précisément les attendus en matière de qualité du tri à effectuer. De nombreux exemples montrent également la solidarité importante de la population et des entreprises à travers la mise à disposition de terrains comme zone de stockage temporaire, à travers la disponibilité de main d'œuvre supplémentaire (EPA, 1995; Reinhart \& McCreanor, 1999).

L'analyse de la littérature a permis de dégager quelques constantes sur les caractéristiques du gisement de déchets post-ouragan, et sur les opérations de gestion que l'on retrouve en partie dans le cas du suivi des déchets post-Irma à Saint-Martin.

\section{Les déchets post-Irma à Saint-Martin : entre insularité et autonomie administrative}

L'île de Saint-Martin dans les Petites Antilles, grande de $87 \mathrm{~km}^{2}$, est divisée en deux parties (française et hollandaise). Officiellement 35600 habitants vivent côté français au $1^{\text {er }}$ janvier 2013 et 73000 sur toute l'île (INSEE et al., 2016). L'ouragan Irma frappe l'île le 6 septembre 2017. Il a alors atteint la catégorie 5, la plus haute sur l'échelle de Saffir/Simpson. Les vents moyens sont d'environ $295 \mathrm{~km} / \mathrm{h}$. L'île est soumise à des pluies intenses, le littoral est frappé par des vagues de 4 à $6 \mathrm{~m}$ et la marée de tempête provoque des submersions marines sur les côtes. La surcote est évaluée à plus de $3 \mathrm{~m}$ en certains points du littoral (Meteo France, 2017). Le bilan humain officiel d'Irma est de onze morts à Saint-Martin. Irma a été suivie dans le même mois de septembre 2017 de José et de Maria, deux ouragans qui n'ont pas frappé directement Saint-Martin mais ont cependant aggravé les dommages sur l'île.

Ces catastrophes récurrentes ont notamment pour impact une production importante de déchets post-catastrophe. Le caractère insulaire impose alors une gestion particulière de la crise, ne permettant pas de s'appuyer sur des territoires ressources voisins. La végétation tropicale de l'île amène également à une production de déchets assez spécifiques. Enfin, l'autonomie administrative dont jouit la « collectivité de Saint-Martin » engendre également des jeux d'acteurs particuliers.

\subsection{Saint-Martin : Identification des quantités de déchets produits par l'ouragan de 2017}

Nous avons observé dans la partie précédente l'importance de connaitre et d'estimer rapidement les gisements de déchets produits par un ouragan. Le retour d'expérience de la gestion des déchets d'Irma, réalisé par l'association Robin des Bois dans le cadre de l'ANR DéPOs permet d'avoir une vue d'ensemble de cette production. Une part importante des gisements a été identifiée sur l'unique site autorisé à recevoir ces déchets en destination finale, la décharge (ISDND - Installation de Stockage des Déchets Non Dangereux) de Grandes Cayes. Le reste du gisement est quant à lui dispersé vers des exutoires informels, cette fois-ci plus complexes à mesurer.

\subsubsection{La décharge de Grandes Cayes : l'unique lieu où identifier les gisements de déchets}

La décharge de Grandes Cayes, unique décharge contrôlée de l'île, a déjà reçu les déchets du cyclone Hugo en 1989. Actuellement administré par l'entreprise Verde SXM, le site est jouxté d'un centre de tri, de transfert, de regroupement et de compostage des déchets : 1'éco-site. Avant même de recevoir les déchets d'Irma, le site a été endommagé par l'ouragan. Le broyeur, la presse à ferraille et la presse à balles de l'éco-site ont été détériorés et la route d'accès submergée et recouverte de plusieurs mètres de sable. En novembre 2018, la presse à balle carton et plastique et le broyeur à verre étaient toujours en attente de réparation.

Le 13 septembre 2017, soit 7 jours après la catastrophe, les déchets sont de nouveau admis dans la décharge, avec une priorité pour les ordures ménagères de routine qui continuent d'être produites, afin d'éviter tout risque sanitaire. Très rapidement, les déchets post-Irma affluent. Du 23 au 31 octobre 
2017, 1096 camions de 15 à $20 \mathrm{~m}^{3}$ sont enregistrés à l'entrée de la décharge ainsi que 3300 petits porteurs ${ }^{16}$. Les déchets provenant des 11 installations temporaires de transit sont déversés à l'extérieur des emprises réglementaires de l'ICPE (Installation Classée pour la Protection de l'Environnement) entre la nouvelle et l'ancienne route d'accès. Une autre zone d'entreposage est ouverte en haut de la plage pour des ferrailles, des déchets verts triés et quelques VHU (Véhicules Hors d’Usages).

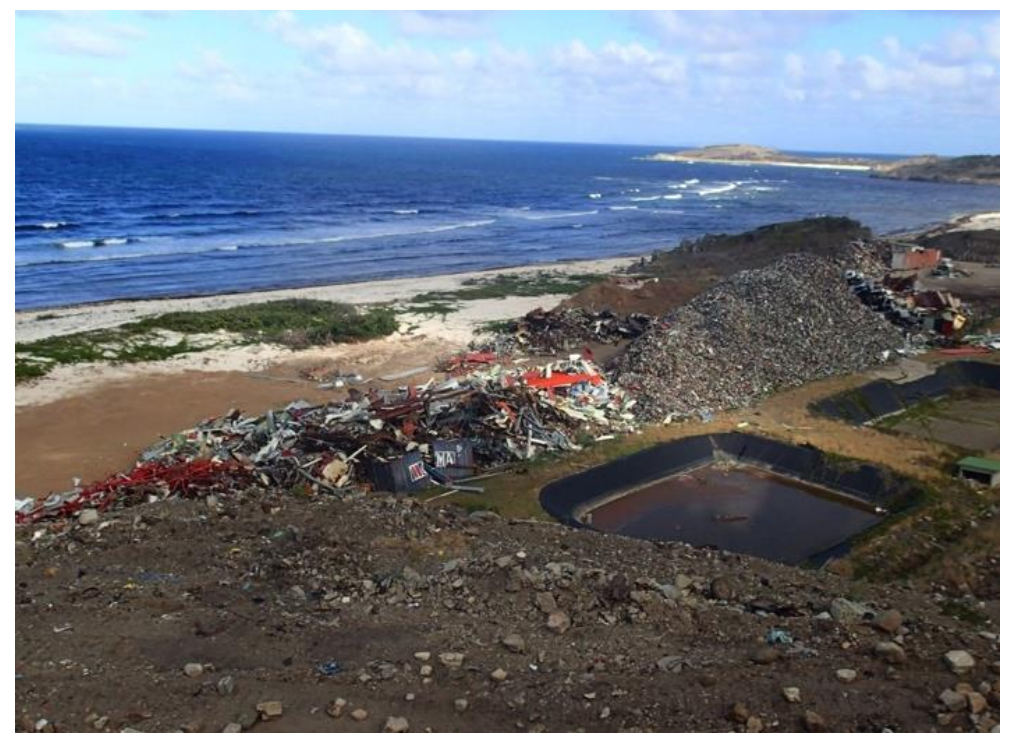

Photo 1. Zone d'entreposage de haut de plage photographiée depuis l'ISDND @ Robin des Bois - ANR DéPOs, 2018

\subsubsection{Déchets ménagers, déchets recyclables : reprendre l'activité de routine au plus vite}

La quantité totale de déchets post-Irma admise entre le 7 septembre 2017 et le 30 juin 2018 à Grandes Cayes est estimée fin juillet par l'exploitant à 55889 tonnes soit 16 mois de déchets réceptionnés en temps normal. Selon la Collectivité, fin juin 2018, 82560 tonnes de déchets post-Irma ont été évacués du domaine public et privé, VHU compris ${ }^{17}$.

\begin{tabular}{|c|c|c|c|}
\hline \multicolumn{1}{|c|}{} & $\begin{array}{c}\text { Tonnage théorique } \\
\text { hors ouragan }\end{array}$ & $\begin{array}{c}\text { Quantité réelle reçue } \\
\text { sur site }\end{array}$ & $\begin{array}{c}\text { Différence attribuée } \\
\text { à Irma }\end{array}$ \\
\hline $\mathbf{2 0 1 7}$ & $41716 \mathrm{t}$. & $85238 \mathrm{t}$. & $43522 \mathrm{t}$. \\
\hline Janvier à juin 2018 & $33224 \mathrm{t}$. & $20858 \mathrm{t}$. & $12366 \mathrm{t}$. \\
\hline Total & $74940 \mathrm{t}$. & $106096 \mathrm{t}$. & $55889 \mathrm{t}$. \\
\hline
\end{tabular}

Tableau 4. Estimation des tonnages des déchets d'Irma réceptionnés sur Grandes Cayes (hors VHU) (Source Verde SXM)

La collecte sélective et le centre de tri (éco-site) ont par ailleurs été perturbés par les arrivées massives de déchets et les difficultés de réparation du matériel endommagé. Les déchets d'Irma pouvant faire l'objet d'une valorisation matière (emballages plastiques, papier-carton, verre) n'ont pas été triés et sont dans les premiers mois partis directement dans la décharge.

Les déchets verts représentent également un gisement notable. Lors du cyclone Gonzalo en 2014, le volume de déchets verts acheminés vers Grandes Cayes avait été de 2000 à 2500 t. Fin mai 2018, le tonnage de déchets végétaux post-Irma était inférieur selon des premières estimations. Les volumes ont

\footnotetext{
${ }^{16}$ Informations collectées sur le terrain par l'association Robin des bois en mai 2018.

${ }^{17}$ Collectivité de Saint-Martin, communiqué du 17 septembre 2018 sur l'organisation des prestations de nettoyage en post-événement.
} 
été en partie réduits par les brûlages autorisés ou tolérés. Les déchets verts acheminés chez Verde SXM sont broyés, mélangés avec des boues de stations d'épuration et mis en fermentation. Le compost vendu à des pépiniéristes avant Irma est actuellement utilisé pour revégétaliser le site de la décharge.

\subsubsection{Les matériaux de construction : l'essence même des déchets post-catastrophe}

Au-delà des déchets ménagers qui continuent à être produits, les déchets post-catastrophe sont en règle générale relativement proches de la nature des déchets de la construction. A Saint-Martin, 19,7\% des bâtiments ont été totalement détruits ou sévèrement endommagés par Irma ${ }^{18}$. Fin juillet 2018, $22 \%$ des bâtis restent à démolir et 20\% impliquent d'importants travaux (Lalere.fr \& AFP, 2018). Il n'y a pas de filière organisée et contrôlée de gestion des déchets de construction, de démolition et des travaux publics. Deux entreprises réalisent du concassage sans les autorisations nécessaires. La tendance générale est au grignotage des étangs et au comblement des rives par ces déchets. Les entreprises sont régulièrement appelées à transporter leurs gravats et déchets de chantier vers l'éco-site. Les appels à respecter les filières réglementaires ne sont pas toujours entendus comme le prouvent les nombreuses décharges illégales de gravats au bord des routes. A titre d'exemple, au rond-point d'Agrément, un site de déversement non autorisé des déchets de démolition post-Irma (gravats, vitres brisées, VHU incendiés ...) occupe une superficie d'environ $6000 \mathrm{~m}^{2}$. Le tas le plus élevé est haut de 5 à $6 \mathrm{~m}$. Les gravats propres peuvent en théorie, après concassage, être utilisés en sous-couches des nouvelles constructions, sous réserve d'acceptabilité par les maîtres d'œuvre et d'ouvrage.

Au-delà des gravats, les déchets de la construction sont également largement constitués de tôles métalliques, traditionnellement utilisées pour les toitures (aluminium, acier inoxydable, acier galvanisé, acier prélaqué, zinc). Certaines entreprises en charge de l'évacuation des déchets vers les sites de transit ont spontanément réalisé un pré-tri des tôles. Environ 4500 t. ont ainsi été acheminées sur le site Grandes Cayes, déjà séparées des autres déchets. Le reste du gisement de métaux ferreux et nonferreux y est arrivé mélangé. Leur extraction des tas d'encombrants a débuté fin juillet 2018 ; il y avait ainsi selon les estimations de Verde SXM 7500 t de tôles à recycler. Les tôles sont broyées avant exportation.

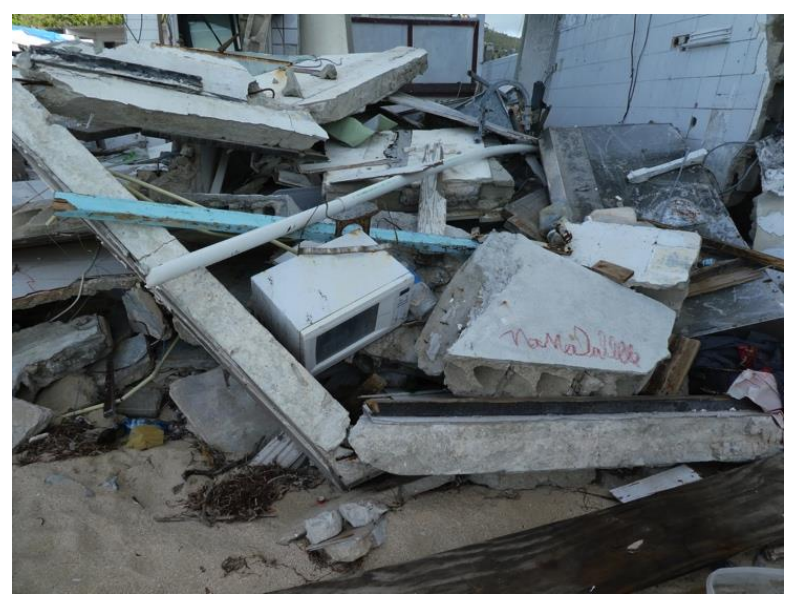

Photo 2 . Béton, plâtre et déchets divers à proximité d'une maison détruite, Grand-Case

C Robin des Bois - ANR DéPOs, 2018

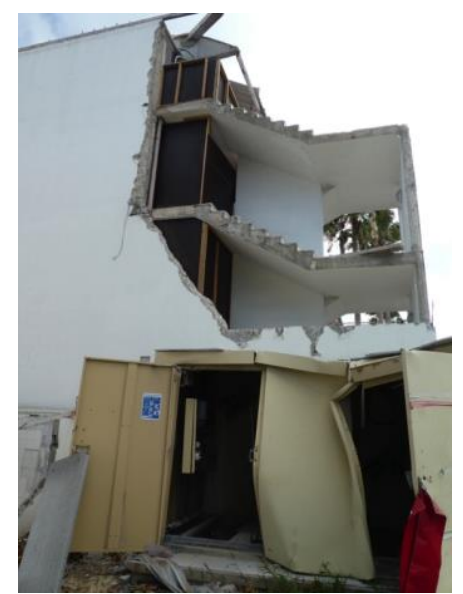

Photo 3. Bâtiment à démolir, Morne Rond (C) Robin des Bois - ANR DéPOs, 2018

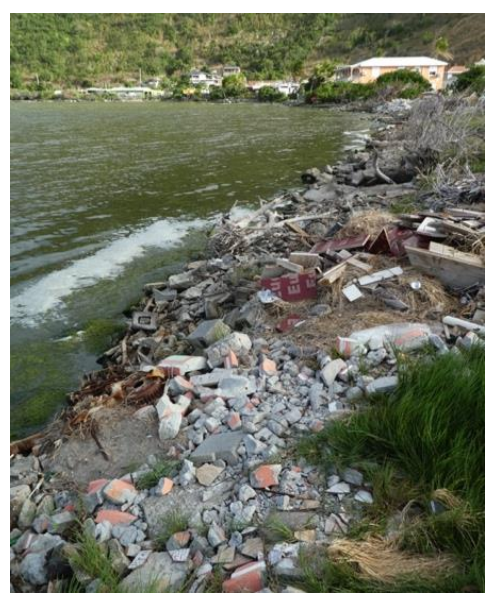

Photo 4. Etang de l'aéroport (C) Robin des Bois - ANR DéPOs, 2018

\footnotetext{
${ }^{18}$ Programme spatial européen Copernicus : https://www.gouvernement.fr/etat-des-lieux-depuis-irma, consulté en novembre 2018 
La gestion d'un certain nombre de déchets est théoriquement très encadrée par des filières de Responsabilité Élargie du Producteur (REP) ou le producteur du produit est responsable de son élimination, soit directement, soit à travers un éco-organisme. Quoiqu'ayant l'obligation de prendre en charge les déchets de situation exceptionnelle, dans les faits, ces filières sont inégalement opérationnelles à Saint-Martin.

Les DEEE (Déchets d'Équipements Électriques et Électroniques), organisés selon une filière REP depuis 2006, sont sous la responsabilité des éco-organismes (structures regroupant tous les producteurs et importateurs d'équipement électriques et électroniques). Saint-Martin dépend en cela de la Guadeloupe. Les éco-organismes sont alors chargés de reprendre gratuitement les DEEE endommagés par des catastrophes naturelles, dès lors qu'ils ont été extraits et triés. C'est ce dernier point qui pose difficulté, car ils n'interviennent qu'à partir d'un tonnage suffisant rassemblé dans un même endroit. Quelques dizaines de tonnes de DEEE pré-triés ou extraits par Verde SXM des tas d'encombrants étaient regroupés à Grandes Cayes en juillet 2018. Selon 1'exploitant, quelques dizaines de tonnes supplémentaires pourront être extraites. Les DEEE en bon état sont expédiés par voie maritime chez AER (Antilles Environnement Recyclage) en Guadeloupe pour dépollution et extraction des parties valorisables, en particulier des métaux ferreux et non-ferreux. Les DEEE sévèrement endommagés ou brûlés sont assimilés à de la ferraille et broyés. Enfin, un grand nombre de DEEE ont été happés par le flux destiné à l'enfouissement. Les panneaux photovoltaïques endommagés à Saint-Martin ne font quant à eux pas l'objet d'un tri spécifique malgré les composants toxiques qu'ils sont susceptibles de contenir.

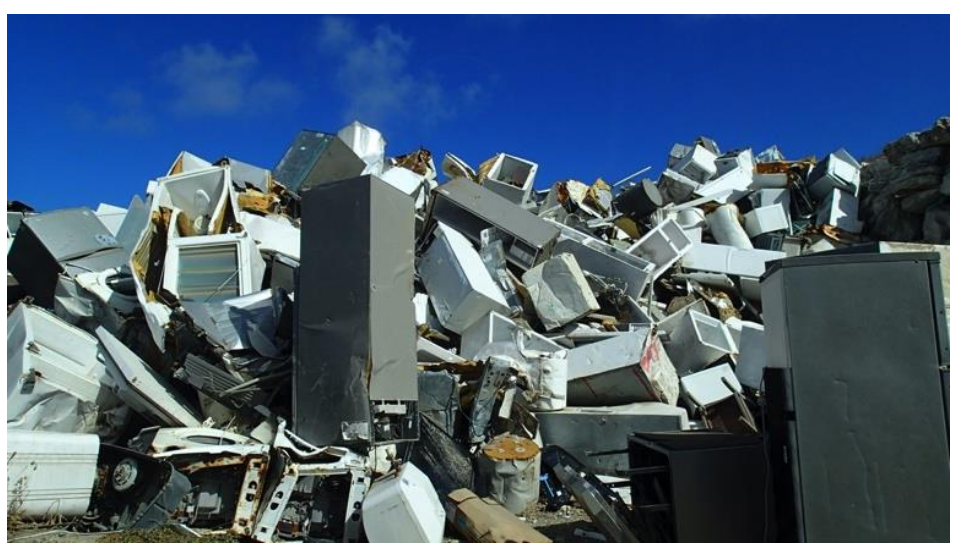

Photo 5. Eco-site de Grandes Cayes (C) Robin des Bois - ANR DéPOs, 2018

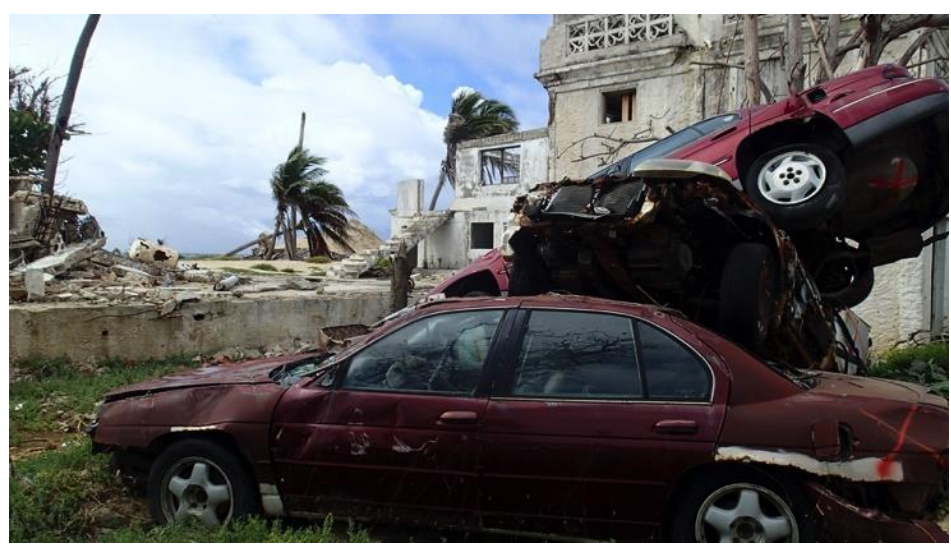

Photo 6. Entrée d'Oyster Pond (C) Robin des Bois ANR DéPOs, 2018

Le traitement des VHU (Véhicules Hors d'Usage) est pour sa part directement de la responsabilité de démolisseurs et broyeurs agréés. Avant même Irma, il y avait sur le territoire de Saint-Martin environ 2000 VHU abandonnés ${ }^{19}$, datant souvent des ouragans antérieurs. À partir de novembre 2017, la collectivité met en place plusieurs opérations d'enlèvement gratuit des véhicules endommagés par l'ouragan. En juillet 2018, 1950 VHU sont arrivés sur l'éco-site, alors seul site agréé pour leur dépollution et démontage. Ce gisement peut permettre de revendre jusqu'à $1500 \mathrm{t}$ de métaux mais génère également une quantité importante de déchets dangereux (Deloitte Développement durable et al., 2018). D'autre part, les VHU «historiques » ne sont pas démontés sur l'éco-site. Ils sont sommairement dépollués et envoyés dans un broyeur, puis sur la table de tri, en fonction depuis mijuillet 2018. Les fractions métalliques, l'aluminium et le cuivre sont séparés des Résidus de Broyage Automobile (RBA) mis en décharge. Les fractions valorisables du broyat et les balles de métaux issus

${ }^{19}$ Entretien avec la Collectivité de Saint-Martin, 2018 
du compactage sont destinées à l'exportation par navires. $3000 \mathrm{t}$ de ferraille (pas seulement issue des VHU) étaient en attente d'exportation en novembre 2018. Depuis mi-septembre, deux garages ont obtenu un agrément «Centre VHU » afin de concourir au démontage des VHU avant leur envoi sur l'éco-site. Les experts en assurance ont estimé le nombre de voitures sinistrées par Irma à environ 4000 (Amblard, 2018). Le différentiel avec les 1950 VHU réceptionnés sur l'éco-site en juillet 2018 s'explique notamment par le nombre de véhicules encore à l'abandon ou regroupés chez des garagistes, par les épaves roulantes toujours en circulation, ou encore les vols et reventes côté néerlandais. Étant donné les nombreuses «anomalies» concernant les immatriculations, les assurances, le transfert transfrontalier et inter-îles des déchets, le bilan exact des VHU Irma restera approximatif.

Enfin, la dernière filière massive sur un tel territoire insulaire concerne les Bateaux de Plaisance Hors d'Usage (BPHU). Créée par le loi de transition énergétique de 2016, cette filière REP n'est pas encore opérationnelle en France métropolitaine et à Saint-Martin. Elle le sera courant 2019. Avant l'arrivée d'Irma, un nombre inconnu d'épaves, remontant notamment à l'ouragan Luis, restait dans les fonds du Grand Étang de Simsonbaai, plus connu sous le nom de lagon de Simpson Bay. En 2012, l'association Métimer avait recensé au moins 26 bateaux coulés, échoués ou abandonnés dans la partie française du lagon. Le nombre de bateaux endommagés ou détruits par Irma côté français est estimé dans les semaines qui suivent la catastrophe entre 500 et 2000 selon les sources. Les épaves les plus dangereuses sont relevées par les militaires du Tonnerre. Le relevage des autres épaves, grâce au support d'un ponton-grue, commence à partir de décembre 2017. Les bateaux assurés sont réparés sur place ou expédiés par barge à l'étranger ou en France métropolitaine. 650 dossiers d'assurance sont connus de Métimer en décembre 2018. En juin 2018, il reste 144 épaves sur toute la partie française de l'île, dont 97 dans le lagon (estimation de la DREAL, la collectivité et l'association Métimer). Seules 17 arborent un pavillon français. Près d'un an après Irma, les procédures de déchéance de propriété des navires abandonnés s'achèvent. Elles sont nécessaires sauf lorsque l'épave présente un danger pour la navigation. Si le propriétaire est identifié, il est mis en demeure d'enlever son épave, ce qu'il n'a pas toujours les moyens ou la volonté de faire. Comme pour les VHU, les BPHU abandonnés sont à la charge des pouvoirs publics. Dans le cas du Victoria, encastré dans le salon d'une maison de Sandy Ground, le propriétaire vénézuélien est introuvable. D'autres bateaux sont revendus à très bas prix entre les différents îles de la région, ou volontairement coulés en mer. En terme de bilan déchets et matières valorisables, les BPHU doivent être considérés comme des maisons ou des mobil-homes flottants, motorisés, auxquels s'ajoutent des équipements électriques et électroniques de navigation et pour certains, de loisirs, des voiles, des câbles et cordages, des engins de secours, souvent des engins de pêche, des équipements de sécurité, des batteries, des hydrocarbures et des fusées de détresse. 


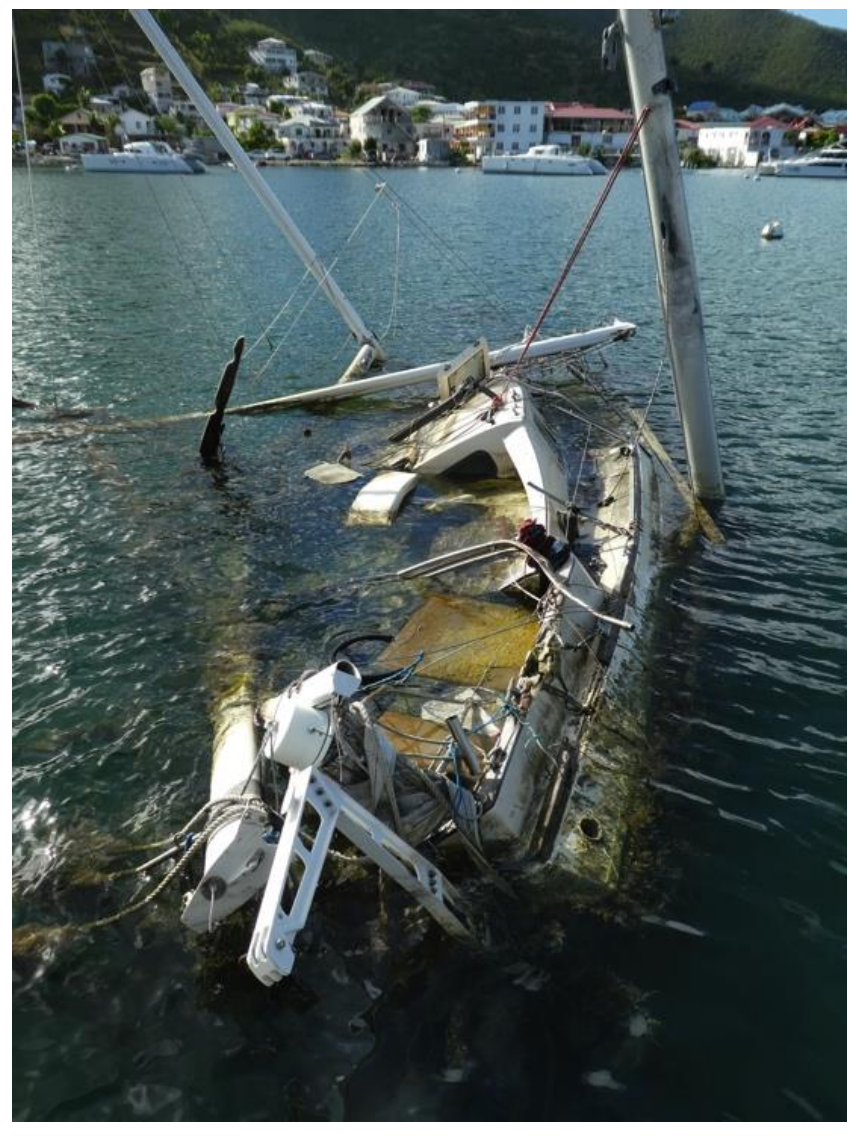

Photo 7. Marina Port-La-Royale (lagon côté français) (C) Robin des Bois - ANR DéPOs, 2018

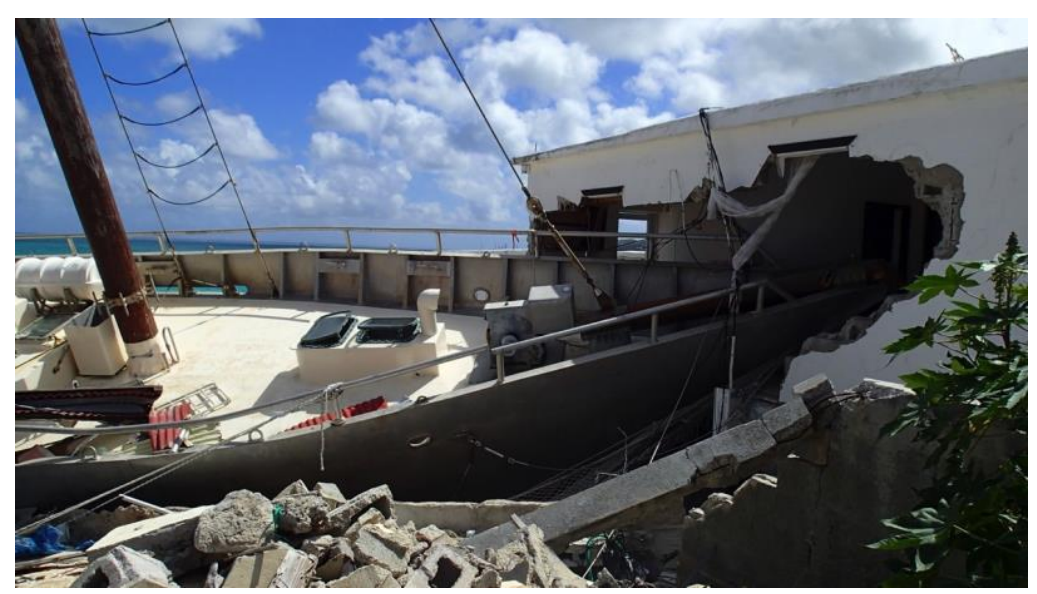

Photo 8. Bateau Victoria, quartier de Sandy Ground (C) Robin des Bois - ANR DéPOs, 2018

\subsubsection{Déchets dangereux : de faibles quantités pour un risque maximum}

La difficulté récurrente de la gestion des déchets dangereux est que, malgré leur caractère dangereux, ils ne sont pas présents en grandes quantités et sont souvent difficiles à repérer sans tri efficace en amont. C'est par exemple le cas des fusées de détresse issues des bateaux. Malgré l'importance de la filière nautique, la filière déchets pyrotechniques n'est pas structurée. Les départs de feu dans les alvéoles de la décharge de Grandes Cayes à cause des fusées de détresse enfouies avec les déchets ménagers étaient globalement en diminution depuis 2 à 3 ans, mais sont en recrudescence depuis Irma.

Les déchets dangereux ne font pas non plus l'objet d'une filière de collecte et de traitement structurée sur l'île, déjà avant le passage de l'ouragan Irma. Les pots de peinture, solvants, emballages contenant des résidus de produits toxiques, les produits chlorés pour piscines, les pesticides périmés et les emballages, entre autres, partent en décharge. Seules les huiles de moteurs usagées sont collectées depuis 2016. En 2017, 110 t ont ainsi été expédiées près du Havre et quatre citernes en 2018.

L'ouragan a également produit son lot d'animaux morts (au moins 7 bovins, 180 caprins et 2500 volailles). Une épidémie de maladie de Carré s'est développée après Irma dans la population canine. En l'absence d'une filière d'équarrissage, les cadavres provenant des élevages détruits ont été saupoudrés de chaux et acheminés vers la décharge de Grande Cayes. Dans un deuxième temps, ils ont été transportés en camion frigorifique et enfouis sans chaux pour favoriser une décomposition rapide. 


\subsection{Jeux d'acteurs et enjeux de territoire au cœur de la gestion des déchets post- catastrophe}

L'identification des gisements de déchets produits par le passage de l'ouragan Irma à Saint-Martin en 2017, réalisée dans le chapitre précédent, a permis de montrer le grand niveau d'incomplétude de ces informations. C'est notamment dû au fait qu'une part importante de ces gisements soient en réalité abandonnés dans la nature ou gérés de façon informelle. Les limites de l'entrée par la typologie de déchets imposent donc de développer d'autres approches. Celles-ci se centrent sur les territoires ayant reçu les déchets, ainsi que sur les acteurs les ayant travaillés.

\subsubsection{Nettoyer l'île : une gestion multi-acteurs}

En l'absence d'identification de l'intégralité des gisements de déchets, le suivi des opérations de nettoyage permet de mieux en comprendre leur gestion. Irma laisse derrière lui une île jonchée de déchets et débris. Sur les voieries gisent principalement des troncs d'arbres et autres déchets végétaux, des poteaux, des câbles électriques, des VHU, des tôles, du sable et de la boue. Dans certaines rues, le sable atteint $2 \mathrm{~m}$ d'épaisseur ${ }^{20}$. Les premières opérations de dégagement des routes sont effectuées par des militaires de la sécurité civile et des sapeurs-sauveteurs pré-positionnés à Saint-Martin ou convoyés sur l'île après l'ouragan. Le bâtiment de la Marine Nationale Tonnerre arrive en renfort le samedi 23 septembre avec 528 militaires, une centaine de véhicules, 1200 tonnes de fret et de matériel de travaux publics. Les déchets sont jusqu'alors poussés sur le bas-côté des routes et chemins. Les équipes de la sécurité civile effectuent, lorsque cela est possible, un pré-tri pour isoler les déchets biodégradables (surtout les déchets verts). La collectivité de Saint-Martin mobilise une trentaine d'entreprises locales pour participer au déblaiement et commencer l'évacuation des déchets vers l'unique site de traitement côté français : la décharge et l'éco-site de Grandes Cayes. Dans certains quartiers isolés, la population réalise ces opérations par ses propres moyens.

Certains habitants commencent les opérations de nettoyage de leur logement ou lieu de travail dès le lendemain du passage de l'ouragan. Plus de deux semaines après, l'Agence Régionale de Santé diffuse en langue française les «Bonnes pratiques et consignes sanitaires après un phénomène cyclonique ». La présence de déchets, de débris, d'objets coupants et tranchants est soulignée et donc la nécessité de «porter des gants et des bottes lors de vos travaux de nettoyage et de déblayage ». Le port effectif de ces Équipements de Protection Individuels est limité aux personnes qui en disposaient avant l'alerte ouragan ou à celles qui ont pu s'en procurer une fois les voies rétablies et les magasins rouverts. Les déchets issus du nettoyage des maisons, appartements, commerces, voies privées, jardins, garages et appentis sont évacués sur des aires de dépose réalisées spontanément par les populations sinistrées au plus près du bâti endommagé21. Ces déchets sont des mobiliers endommagés et/ou mouillés (lits, matelas, tables, chaises, canapés...), de Déchets d'Equipements Electriques et Electroniques (DEEE : réfrigérateur, climatiseurs, jeux vidéo...) et divers autres déchets ménagers et assimilés (produits d'entretien et d'hygiène, médicaments, phytosanitaires, livres ...).

\footnotetext{
${ }^{20}$ Entretien avec M. Armengaud Régis, Préfecture de Saint-Barthélemy et de Saint-Martin, Chef du Service des Territoires et de la Mer et du Développement Durable, le 31 mai 2018.

${ }^{21}$ Arrêté du 30/07/12 relatif aux prescriptions générales applicables aux installations classées soumises à déclaration sous la rubrique $\mathrm{n}^{\circ} 2719$ (installation temporaire de transit de déchets issus de pollutions accidentelles marines ou fluviales ou de déchets issus de catastrophes naturelles).
} 


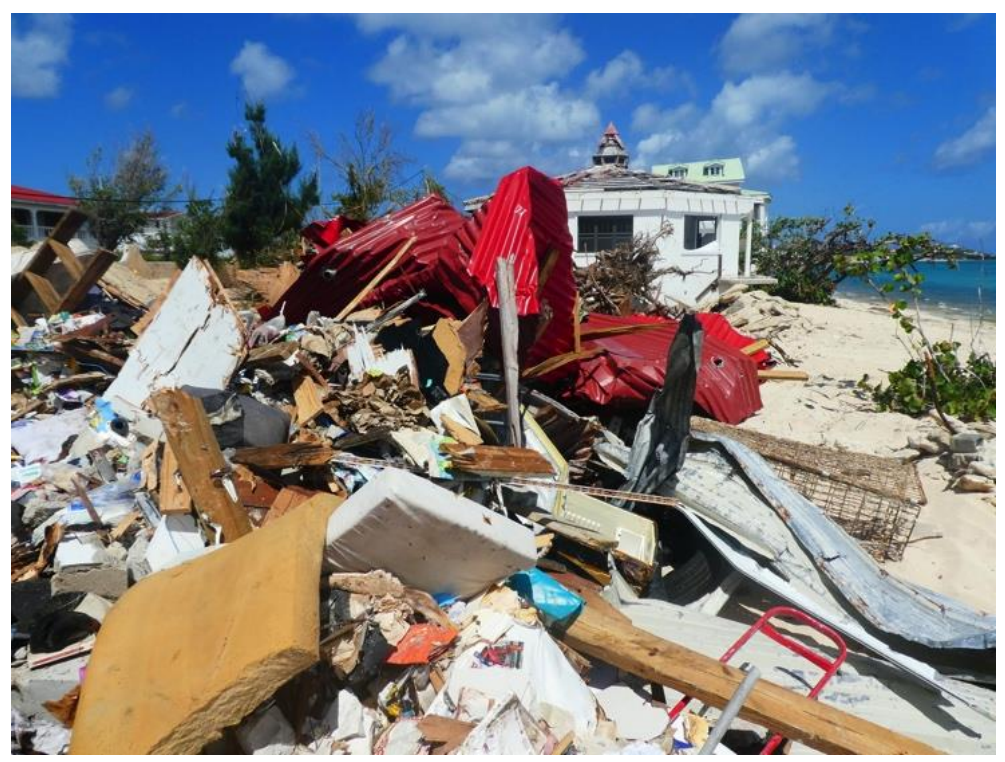

Photo 9. Aire de dépose à Sandy Ground : mélange de matelas, matériels scolaires, livres, tôles, volets, plastique, bois, magazines, ferraille, carton... (C) Robin des Bois - ANR DéPOs, 2018

Un pré-tri des déchets végétaux, des tôles et des gravats est effectué sur certaines aires de dépose. Des déchets dangereux (solvants, bouteilles de gaz, aérosols...) sont mêlés au gisement de déchets dits « encombrants », un terme souvent utilisé par les intervenants publics et privés pour les déchets postcatastrophe en mélange, synonyme de «tout-venant». Les encombrants ne se rattachent à aucune nomenclature réglementaire. Du 10 mai au 15 juillet 2018 une grande campagne de nettoyage est organisée par la collectivité qui tente de mettre fin aux dépôts sauvages de déchets dans les rues et dans la nature en mettant à disposition des bennes dans les différents quartiers et en rendant l'accès à la décharge de Grandes Cayes gratuit. Du 20 novembre au 20 décembre 2018, des bennes de $20 \mathrm{~m}^{3}$ sont de nouveau mises à disposition. Il est demandé au public de trier les déchets verts et les tôles. Des dépôts autour des bennes de déchets mêlés sont encore déplorés plus d'un an après Irma. Les opérations de nettoyage se poursuivent encore aujourd'hui au gré du retour sur l'île des habitants évacués après l'ouragan. Elles mettent parfois à jour des gisements de déchets putrescibles infestés et nauséabonds.

Les flux de déchets de routine, c'est-à-dire des déchets qui auraient été produits même en l'absence de l'ouragan, ne rentrent pas dans le bilan des déchets Irma. Cependant, les flux Irma/non-Irma n'ont pas toujours été séparés; dans les premiers jours, des déchets putrescibles sont jetés dans les rues ce qui favorise la prolifération de mouches, de rats et d'odeurs pestilentielles. Le 7 septembre au matin, les camions de collecte, appartenant à des entreprises privées, sont hors service. Trois stations-services rouvrent rapidement et les rotations de camions opérationnels reprennent à partir du mercredi 13 septembre, une fois la décharge accessible. La collecte des déchets est aussi perturbée par la perte ou l'endommagement de bacs-poubelles qui n'avaient pas été mis en sécurité lors de l'alerte ouragan. La collectivité annonce l'installation de 420 nouveaux bacs entre décembre 2017 et janvier 2018 ${ }^{22}$. Le service normal de ramassage des déchets et encombrants reprend le 7 octobre 2017, soit un mois après le passage d'Irma et la collectivité appelle la population à ne plus jeter les déchets sur la voie publique et à reprendre le tri sélectif du verre et des cartons. En novembre 2018, les bornes de tri du plastique et du verre emportées ou endommagées par Irma ne sont pas toutes remplacées.

\subsection{2. Établir des installations temporaires de transit pour canaliser les déchets}

Dans l'urgence de la catastrophe, l'une des difficultés est d'identifier des lieux pour déposer, de façon temporaire, les déchets, afin d'éviter tout risque sanitaire et de permettre l'accessibilité aux secours. Saufs apports volontaires vers la déchèterie de Galisbay-Bienvenue, seule déchetterie de l'île,

${ }^{22}$ Collectivité de Saint-Martin, « Nettoyage des sites de stockage des déchets d'Irma », 12 décembre 2017 
ou transfert direct vers l'éco-site et la décharge de Grandes Cayes, les déchets issus du déblaiement des routes et des aires de dépose spontanées sont évacués vers 11 installations temporaires de transit de déchets désignées par la collectivité et ouvertes après géo-référencement et évaluation des risques de pollution $^{23}$ par la Direction de l'Environnement, de l'Aménagement et du Logement - DEAL (figure $\mathrm{n}^{\circ} 1$ ). Il s'agit de terrains de sport, de parkings et de terrains vagues immédiatement disponibles et accessibles.

Les rotations vers les 11 sites sont effectuées jusqu'au 20 octobre 2017. Mi-novembre 2017, la quantité entreposée sur les 11 sites est estimée à $109000 \mathrm{~m}^{3}$ soit environ $27250 \mathrm{t}$ (Daizey, 2017). Le déstockage de l'ensemble des installations temporaires vers Grandes Cayes commence mi-novembre 2017. Jusqu'au mois de décembre 2017, une cinquantaine d'entreprises, 1091 travailleurs, 81 chefs d'équipe et 1000 ouvriers sont mobilisés par la collectivité, pour réaliser près de 300 aller-retours journaliers vers la décharge. Les moyens matériels comprennent 19 pelles, 10 chargeurs, 22 minipelles, 19 tractopelles et 155 camions $^{24}$. Des pierres sont déposées à l'entrée de certains sites après leur déstockage pour éviter qu'ils ne deviennent des décharges sauvages. Certains apports incontrôlés sont cependant toujours déplorés au mois de mai 2018, par exemple sur le stade Albéric Richard.

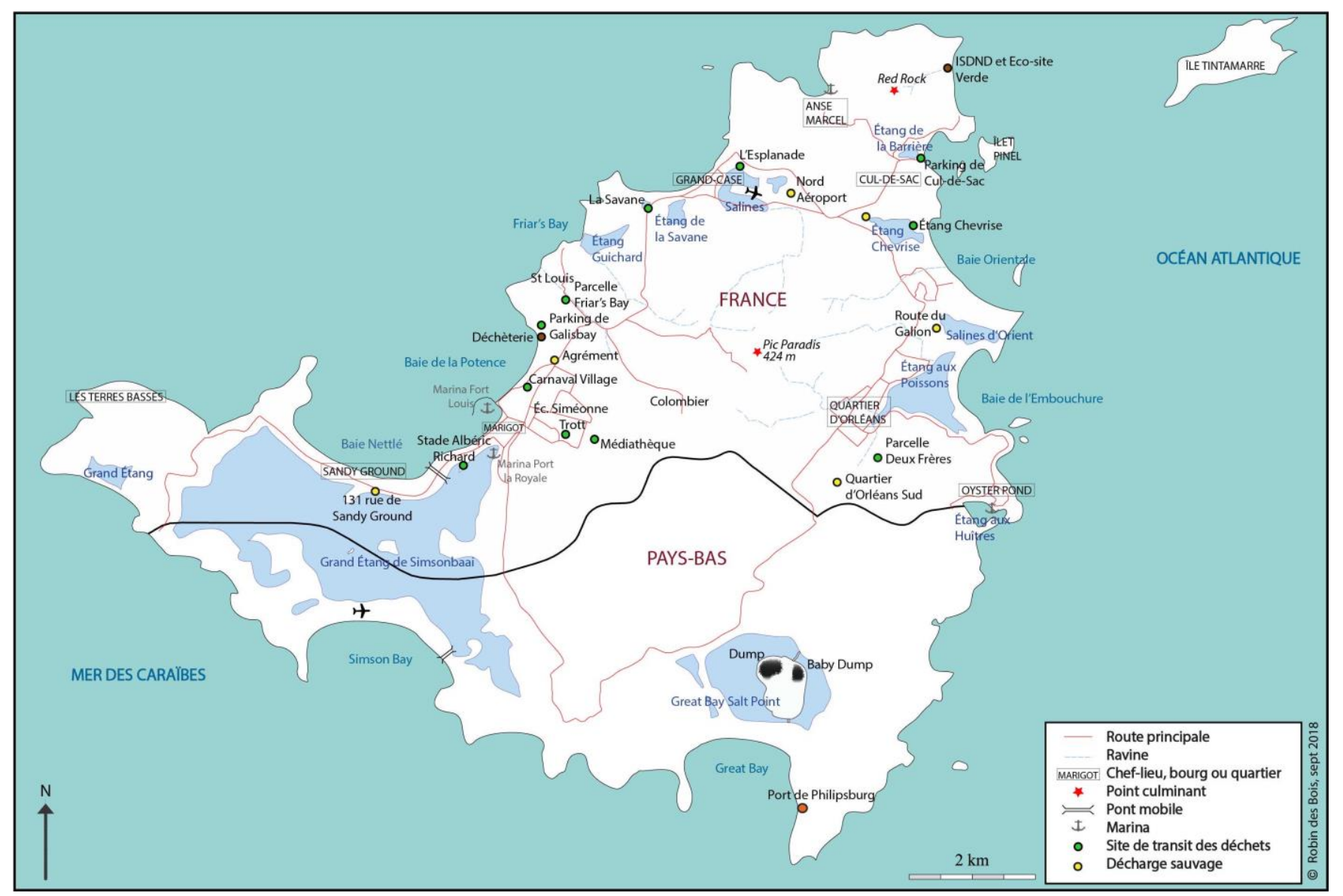

Figure 1. Saint-Martin, localisation des sites de transit et de traitement des déchets et des principales décharges sauvages.

\footnotetext{
${ }^{23}$ Toutefois sans prise en compte des normes ICPE (Installations Classées pour la Protection de l'Environnement) habituellement appliquées. Officiellement, seules les installations temporaires de transit de déchets issus de catastrophes naturelles d'un volume supérieur à $100 \mathrm{~m}^{3}$ sont des Installations Classées (rubrique 2719). Dans les faits, il est difficile d'estimer lorsque ce seuil est dépassé. ${ }^{24}$ Entretien avec Mme Terrac Charlotte, Collectivité de Saint-Martin, Chargée de mission gestion des risques majeurs, 31 mai 2018.
} 
Sans disposer, pour le moment, de chiffre précis ${ }^{25}$ concernant les déchets «évaporés » dans la nature, il est toutefois possible, d'après les observations de terrain (Robin des Bois, 2018), de constater leur grande ampleur.

Les brûlages à ciel ouvert sont la première de ces techniques mises en œuvre pour faire face à une situation d'urgence. Ils ont été autorisés par dérogation du jeudi 21 septembre 2017 au dimanche 24 septembre 2017, de $7 \mathrm{~h}$ à $19 \mathrm{~h}$, selon le sens du vent, en raison des risques sanitaires et environnementaux redoutés (prolifération de moustiques, rats ...). Les prescriptions de l'arrêté $n^{\circ} 2017$ 218/PREF/STMDD ${ }^{26}$ n'ont pas été respectées stricto sensu par les populations et les entreprises désireuses de réduire au plus vite le volume des déchets et d'en supprimer la vision par la combustion. Les brûlages volontaires se sont multipliés sur les parkings, sur les plages et au bord des routes. Au moins une maison, située trop près des bûchers, a pris feu et imposé l'intervention des pompiers ${ }^{27}$. L'inspection des foyers de cendres, qu'ils soient frais ou anciens, montre que des plastiques, des emballages, du mobilier, des éléments métalliques et des pneus ont été brûlés. Des VHU ont également été brûlés. Trois jours après Irma, environ 150 VHU ont pris feu à la sortie de Sandy Ground ${ }^{28}$. Le feu aurait été causé par un court-circuit et s'est propagé de véhicule en véhicule. D’autres incendies ont été déplorés et sont encore redoutés par les autorités locales.

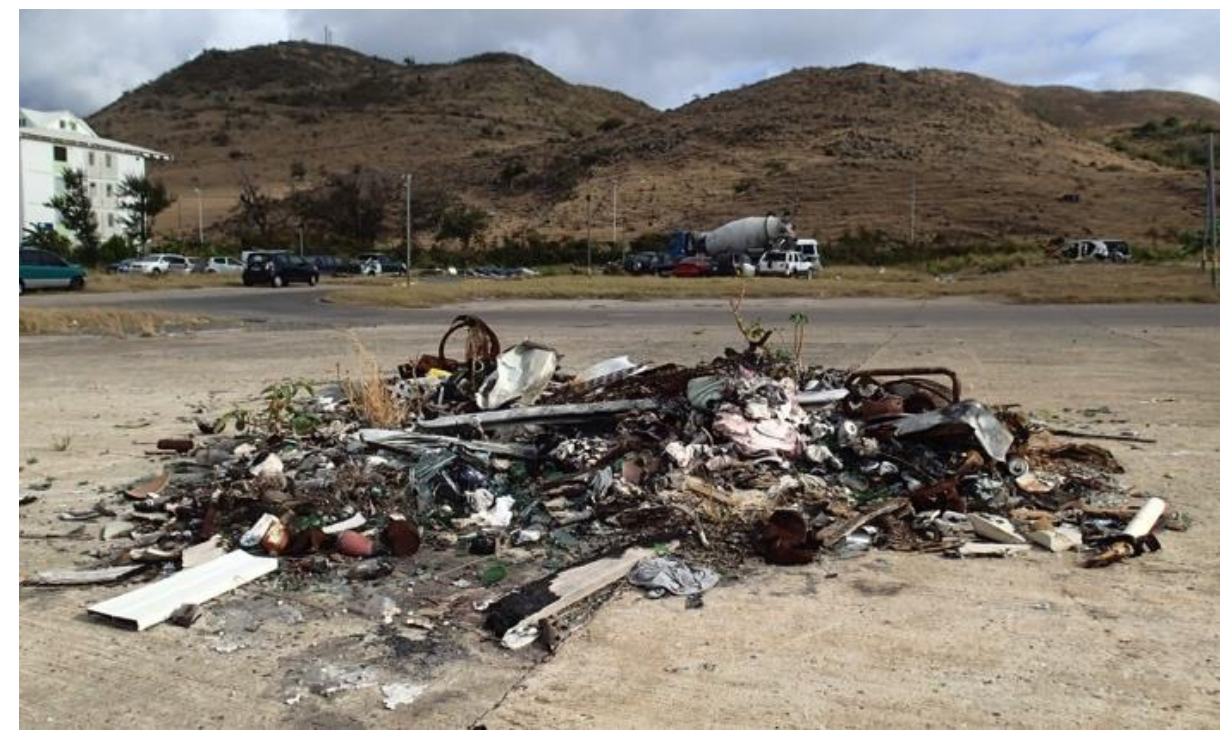

Photo 10. Cul de Sac, résidence Chevrise (C) Robin des Bois - ANR DéPOs, 2018

La géographie de Saint-Martin favorise également la dispersion des déchets en mer et sur terre, au fond des ravines, dans la mangrove, les étangs et la mer. Les déchets les plus lourds se déposent sur les fonds marins et les plus légers sont emportés au large parfois sur de longues distances. Lors du tsunami au Japon en 2011, les autorités avaient estimé que 5 millions de tonnes de déchets étaient partis à la mer, dont 3,5 millions de tonnes auraient coulé rapidement et au moins 1,5 millions de tonnes seraient partis à la dérive dans l'Océan Pacifique, jusqu'en Alaska. Des échouages ont été observés en Alaska (Robin des Bois, 2011a). Des opérations de ramassage de déchets au fond de l'eau ont été réalisées par l'établissement portuaire de Saint-Martin et la collectivité au mois de mai 2018. Des tôles en particulier, mais aussi des bacs roulants à déchets, des bois et des débris divers ont été remontés des

\footnotetext{
${ }^{25}$ Cette quantification est notamment l'un des aspects du projet de recherche DEPOS financé par l'ANR, auquel participent les auteurs de cet article.

${ }^{26}$ Arrêté préfectoral n²017-218/PREF/STMDD du 21 septembre 2017 portant autorisation de brûlage des déchets verts et DIB (bois, cartons, papiers) à l'air libre autorisé de 7 h00 à 19 h00 du jeudi 21 septembre 2017 au dimanche 24 septembre 2017

${ }^{27}$ Entretiens avec les acteurs de terrain, mai-juin 2018.

${ }^{28}$ Entretiens avec Mme Terrac Charlotte, Collectivité de Saint-Martin, Chargée de mission gestion des risques majeurs, les 29 et 31 mai 2018
} 
petits fonds par des plongeurs professionnels. $10 \mathrm{t}$ d'encombrants divers ont ainsi été collectés en face de Grand Case (Le Pélican, 2018).

Dans les milieux naturels terrestres ou semi-aquatiques, des opérations de nettoyage sont régulièrement réalisées. Le collectif Clean Saint-Martin, Sandy Ground on the Move, The Madtwoz Family, le Secours Populaire et Clean St Martin sont quelques-unes des structures qui fédèrent leurs volontaires. Des scouts sont venus prêter main forte. Les sites protégés de la Réserve naturelle de Saint-Martin, les 14 étangs et $11 \mathrm{~km}$ de rivages terrestres du Conservatoire du littoral ont été sévèrement impactés par les déchets d'Irma, en particulier la mare de la Baie Lucas, les étangs de Chevrise, de la Barrière aux Poissons, du cimetière de Grand-Case et de l'aéroport. Les déchets sont dispersés dans les plans d'eau, dans les houppiers, les palétuviers et sur les plages : toitures, tôles, murs, poutres, voitures, bateaux, containers, mobiliers, bidons, matelas, transats, bornes de tri ...(Conservatoire du Littoral, 2018). 1289 t de déchets ont été en deux mois ramassés sur les sites du Conservatoire. Le nettoyage doit sur certains sites être renouvelé en raison des nouveaux apports sauvages de déchets. C'est notamment le cas sur le site du Galion où, de surcroît, des brûlages ont été déplorés sur les dépôts.

Enfin, face au manque d'installations pour réceptionner les déchets (en dehors du site de Grandes Cayes), les décharges sauvages se multiplient également. Les appels post Irma réitérés dans la presse locale (uniquement en français) concernant le délit d'abandon de déchets prévu par l'article L. 541-3 du code de l'environnement et les amendes encourues n'ont pas eu sur les populations multilingues d'effets spectaculaires. Les déchets projectiles propulsés par Irma ont été parfois suivis par des apports anthropiques de déchets selon l'adage " le déchet attire le déchet ». Selon des prospectives optimistes, une partie de ces décharges sauvages devrait-être au final évacuée vers Grandes Cayes par la Collectivité. Certaines d'entre elles dateraient des ouragans antérieurs.

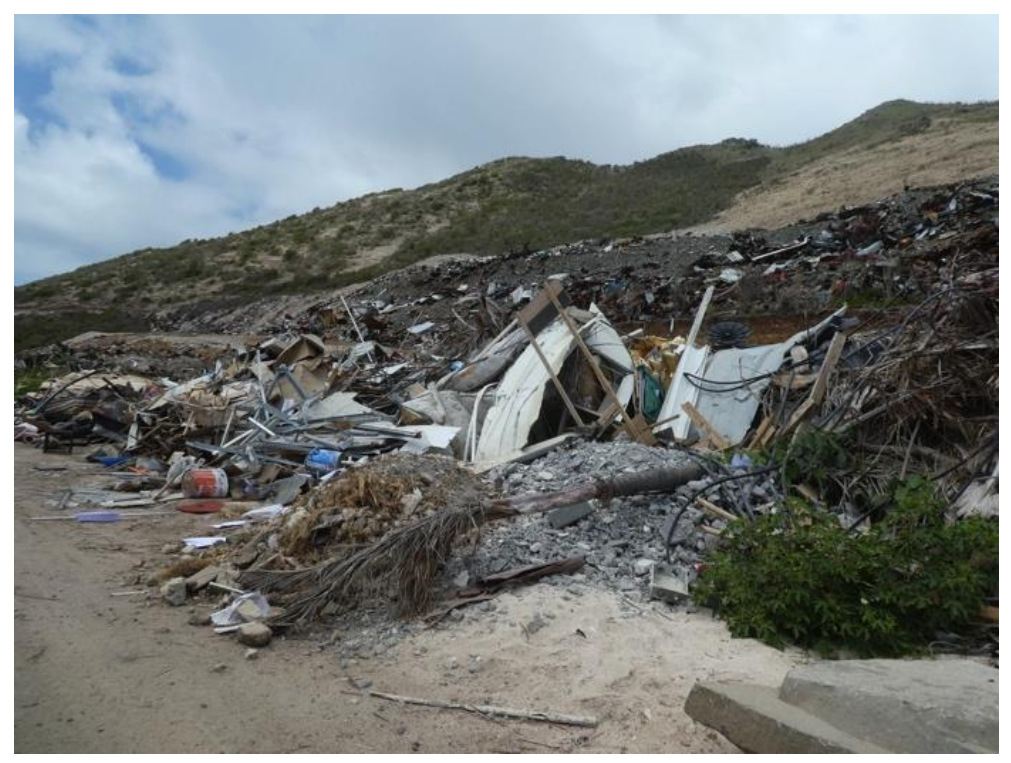

Photo 11. Sur la route d'accès au site de Grandes Cayes, des dépôts sauvages de déchets en mélange (premier plan) s'ajoutent aux dépôts organisés (second plan) @ Robin des Bois - ANR DéPOs, 2018

\subsubsection{Les acteurs informels à ne pas négliger dans le suivi des gisements}

Les retours d'expériences précédents ont montré que, notamment dans des pays en voie de développement, les acteurs informels jouaient un rôle primordial dans la gestion des déchets postcatastrophe. Si Saint-Martin est un territoire français, son isolement géographique semble faire apparaitre des caractéristiques hybrides, avec, comme dans les grandes Antilles voisines (Durand et al., 2015), également une présence importante d'acteurs informels. La récupération et le réemploi de matériaux, d'objets et de pièces détachées ont été largement mis en œuvre de manière formelle ou informelle. Ces éléments se soustraient au bilan déchets d'Irma, sans garantie que leur réemploi soit 
sans risque pour les personnes et l'environnement. A titre d'exemples, des tôles dispersées dans la nature ont ainsi été récupérées pour réparer des habitations qui seront soumises aux futurs évènements climatiques. Les gravats issus des destructions sont rapidement utilisés pour combler toute concavité avant de nouveaux travaux. Le marché formel et informel des pièces détachées de voitures s'est très rapidement développé. Certains VHU sur le domaine public ont été dépouillés de leurs portières, parebrise et autres accessoires. Pendant les 2 mois qui ont suivi l'ouragan, Grandes Cayes a été considéré comme un libre-service de pièces détachées pour Saint-Martin, mais également pour d'autres îles alentour. Des climatiseurs, des réfrigérateurs et des congélateurs ont été détournés de la filière règlementaire DEEE, certains pour être réparés avec des pièces détachées dont l'origine n'est pas toujours connue, d'autres pour être vidangés à l'air libre de leurs fluides frigorigènes afin de revendre la masse métallique.

La décharge de Sint Maarten (la Dump), sur la partie néerlandaise de l'île, a été ouverte dans les années 1970 avec des gravats et des remblais sur la Great Salt Pond, une zone humide et de marais salants d'une superficie approximative de $200 \mathrm{ha}^{29}$. En 1995, la Dump a reçu la quasi-totalité des déchets néerlandais de l'ouragan Luis. Cette décharge qui continue à être exploitée n'a pas pu absorber le flux de déchets d'Irma produits à Sint Maarten et un nouveau site, appelé Baby Dump, a été ouvert à l'Est. L'accès aux décharges est gratuit et des camions venus de la partie française viendraient en profiter. Les incendies volontaires et organisés y sont fréquents. Un récupérateur de métaux équipé d'un broyeur est également implanté dans la zone ; il reçoit des VHU immatriculés en France.

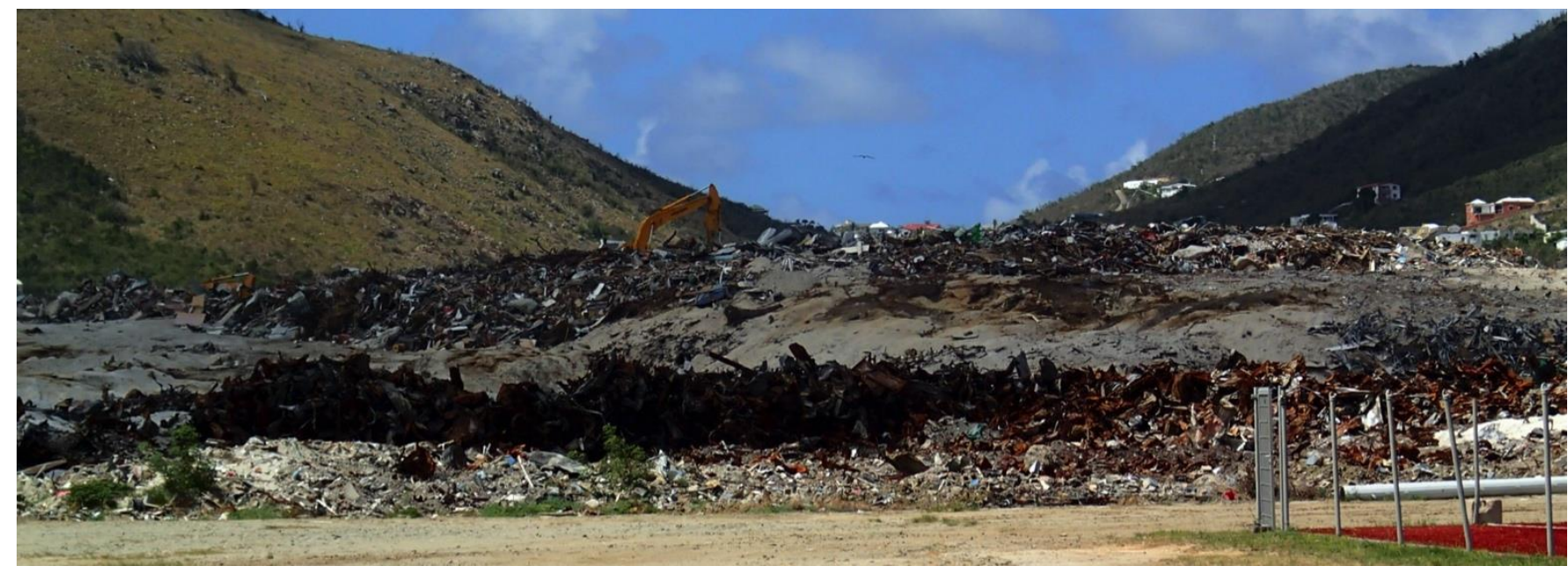

Photo 12. La Baby Dump calcinée (C) Robin des Bois - ANR DéPOs, 2018

\section{Conclusion}

La revue de la littérature et les observations faites à Saint-Martin 8 mois après le passage de l'ouragan Irma (2017) rendent compte des difficultés du suivi des déchets post-ouragan : installation dans l'urgence des aires de stockage temporaire, difficultés à réintroduire certains flux de déchets dans leur filière de gestion habituelle, enfouissement massif, pollutions des eaux, du sol, dépôts sauvages de déchets... Cette difficulté liée aux exutoires est, comme nous l'avions mis en évidence dans la première partie de cet article, le déterminant de l'organisation de la gestion des déchets post-ouragan. Mais plus que sur tout autre territoire, de par sa situation, comme ce qui a été observé sur l'île de Hauai Hawai, la faible disponibilité du foncier, la difficile connection à d'autres territoires ressources, l'impossibilité d'avoir accès à la même offre de filières de traitement et de valorisation que sur le reste du territoire français, rendent la gestion des déchets post-ouragan particulièrement complexe. Par ailleurs, il convient de s'interroger sur les caractéristiques du fonctionnement du service de gestion des déchets, en milieu insulaire, en période normale. La situation en post catastrophe n'est peut-être qu'une version dégradée des difficultés déjà existantes: accès limités aux infrastructures de traitement,

${ }^{29}$ D’après photos aériennes et (Fontan, 2018) 
saisonnalité et nature spécifique du gisement de déchets liées à l'activité touristique (Camilleri-Fenech et al., 2018; Eckelman et al., 2014; Robin des Bois, 2011b). Cet article ouvre donc la voie au projet de recherche DéPOs, visant à mettre en œuvre une méthodologie de quantification et de prise en compte des déchets post-catastrophes, en amont des situations de crise.

\section{Remerciements}

Les auteurs remercient l'Agence Nationale de la Recherche qui soutient le projet de recherche Déchets Post Ouragans - (ANR-18-OURA-0003) et l'ensemble des partenaires de l'ANR DéPOs : Université Paris-Est Marne-la-Vallée, Le Mans Université, Avignon Université, IFSTTAR, Association Robin des Bois et Université de Mons (partenaire international associé).

\section{Bibliographie}

Amblard, F. (2018, 7 avril 2018). Le traitement des épaves : une activité sous haute surveillance. Le Pélican. Retrieved from http://www.lepelican-journal.com/saint-martin/societe/Le-traitement-des-epaves-une-activite-sous-hautesurveillance-15742.html

Aquino, D. H., Wilkinson, S. J., Raftery, G., Potangaroa, R., \& Chang-Richards, A. (2018). Challenges to building housing resilience: the case of Fiji post-cyclone Winston. Procedia Engineering, 212, 475-480. doi:10.1016/j.proeng.2018.01.061

Beraud, H. (2013). Initier la résilience du service de gestion des déchets aux catastrophes naturelles. Le cas des territoires urbains et de l'inondation. (Doctorat sous la direction de), Université Paris Est Marne-la-Vallée,

Beraud, H., Jadot, J., Barroca, B., Hubert, G., \& Bauduceau, N. (2012). Mécadépi. Méthode d'Evaluation et CAractérisation des DEchets Post Inondation. Rapport final. Retrieved from

Boxer, B., \& Oberstar, J. L. (2008). Hurricane Katrina: continuing debris removal and disposal issues. Retrieved from https://www.documentcloud.org/documents/242157-hurricane-katrina-continuing-debris-removal-and.html

Brandon, D. L., Medina, V. F., \& Morrow, A. B. (2011). A Case History Study of the Recycling Efforts from the United States Army Corps of Engineers Hurricane Katrina Debris Removal Mission in Mississippi. Advances in Civil Engineering, 2011,1-9. doi:10.1155/2011/526256

Brown, C. (2012). Disaster waste management: a systems approach. University of Canterbury,

Brown, C., \& Milke, M. (2016). Recycling disaster waste: Feasibility, method and effectiveness. Resources, Conservation and Recycling, 106, 21-32.

Brown, C., Milke, M., \& Seville, E. (2011). Disaster management: A review article. Waste Management, vol. 31, Iss. 6, 1085-1098.

Camilleri-Fenech, M., Oliver-Solà, J., Farreny, R., \& Gabarrell, X. (2018). Where do islands put their waste? - A material flow and carbon footprint analysis of municipal waste management in the Maltese Islands. Journal of Cleaner Production, 195, 1609-1619. doi:10.1016/j.jclepro.2017.07.057

COES. (2005). Training manual. Debris management in disaster recovery. Retrieved from State of California:

Conservatoire du Littoral. (2018). Analyse et diagnostic écologique post cyclone irma 2017 à Saint-Martin. Retrieved from http://base-documentaire.pole-zh outremer.org/documents/Docs_lies/2018/09/18/A1537289696SD_CELRL_ Diag_Ecologique_Post_IRMA_SXM_Rapport.pdf

Crowley, J. (2017). A measurement of the effectiveness and efficiency of pre-disaster debris management plans. Waste Management, 62, 262-273. doi:10.1016/j.wasman.2017.02.004

Daizey, V. (2017, 10/11/2017). Irma, le vertige des chiffres. 97150.

Deloitte Développement durable, Monier, V., Salès, K., Lucet, L., Benhallam, R., Basciano, R., . . . Filmon, K. (2018). Rapport Annuel de l'Observatoire des Véhicules Hors d'Usage. Retrieved from https://www.ademe.fr/sites/default/files/assets/documents/observatoire-vhu-donnees2016-201804-rapport.pdf

Dubey, B., Solo-Gabriele, H. M., \& Townsend, T. (2007). Quantities of arsenic-treated wood in demolition debris generated by hurricane Katrina. Environmental science \& technology, Vol. 41, n5, 1533-1536.

Durand, M., Popescu, R., \& D’Ercole, R. (2015). Les déchets post-catastrophe à Haïti : les jeux d'acteurs d'une gestion informelle. VertigO(Volume 15 Numéro 1). doi:10.4000/vertigo.15970 
Eckelman, M. J., Ashton, W., Arakaki, Y., Hanaki, K., Nagashima, S., \& Malone-Lee, L. C. (2014). Island Waste Management Systems: Statistics, Challenges, and Opportunities for Applied Industrial Ecology. Journal of Industrial Ecology, 18(2), 306-317. doi:10.1111/jiec.12113

EPA. (1995). Planning for disaster debris. Retrieved from https://nepis.epa.gov/Exe/ZyPDF.cgi/ 10000MU0.PDF?Dockey=10000MU0.PDF

EPA. (2008). Planning for natural disaster debris. Retrieved from https://nepis.epa.gov/Exe/ZyPDF.cgi/P1004PRS.PDF? Dockey=P1004PRS.PDF

EPA. (2018). Planning for natural disaster debris. Retrieved from https://www.regulations.gov/contentStreamer? documentId=EPA-HQ-OLEM-2017-0657-0006\&contentType=pdf

Escobedo, F. J., Luley, C. J., Bond, J., Staudhammer, C. L., \& Bartel, C. (2009). Hurricane debris and damage assessment for Florida urban forest. Arboriculture \& Urban Forestry, 35(2), 100-106.

FEMA. (2006). HAZUS MH MR3 Hurricane Model Technical Manual. Retrieved from

FEMA. (2007). Public assistance. Debris management guide. Retrieved from https://www.fema.gov/media-librarydata/20130726-1826-25045-7418/fema 325 debris management guide 2007.07.25.pdf

Fontan, F. (2018, 13 juin 2018). Décharge de St Maarten : " la situation est devenue hors de contrôle depuis Irma". Soualiga Post.com. Retrieved from http://www.soualigapost.com/fr/actualite/22870/d\%C3\%A9chets/d\%C3\% A9charge-de-st-maarten-la-situation-est-devenue-hors-de-contr\%C3\%B4le-depuis-irma

Hassett, W. L., \& Handley, D. M. (2006). Hurricane Katrina: Mississippi's Response. Public works management policy, Vol. 10, $n^{\circ}$ 4, 295-305.

INSEE, Naulin, A., Le Corre, L., \& Servans, G. (2016). Saint-Martin : terre d'accueil et de constrastes. Retrieved from https://www.insee.fr/fr/statistiques/fichier/2527810/ga_ind_10.pdf

La1ere.fr, \& AFP. (2018). Saint Martin : plus de 15\% des bâtiments impropres à l'habitation, selon une étude. Retrieved from https://la1ere.francetvinfo.fr/saint-martin-plus-15-batiments-impropres-habitation-etude-611554.html

Le Pélican. (2018, 4 juin 2018). 10 tonnes de déchets collectés en mer, Saint Martin. Le Pélican. Retrieved from http://www.lepelican-journal.com/saint-martin/environnement/10-tonnes-de-dechets-collectes-en-mer-16030.html

Luther, L. (2008). Disaster debris removal after Hurricane Katrina: status and associated issues. Retrieved from Washington: https://fas.org/sgp/crs/misc/RL33477.pdf

Meteo France. (2017). Passage de l'ouragan exceptionnel Irma sur les îles françaises des Antilles les 5 et 6 septembre 2017. Retrieved from http://www.meteofrance.fr/espace-presse/53976142-passage-de-l-ouragan-exceptionnel-irmasur-les-iles-francaises-des-antilles-les-5-et-6-septembre-2017

Moe, T. L. (2010). Cleanup after Katrina: an analysis on policy, process, priorities, problems, and politics. Disaster prevention et management, vol. 19, 314-332.

Pagney Bénito-Espinal, F., \& Bénito-Espinal, E. (1991). L'Ouragan Hugo: genèse, incidences géographiques et écologiques sur la Guadeloupe. Pointe-à-Pitre: Parc national de la Guadeloupe : Direction régionale des affaires culturelles de la Guadeloupe : Agence guadeloupéenne de l'environnement, du tourisme et des loisirs.

Popescu, R., Durand, M., \& d'Ercole, R. (2014). La gestion des déchets post-catastrophe à Port-au-Prince : entre relégation et proximité. EchoGéo(30). doi:10.4000/echogeo.14070

Ravenet, A., \& Vuillet, J.-L. Dean, le cyclone. Martinique : histoire d'une reconstruction (Orphie ed.).

Reinhart, D. R., \& McCreanor, P. T. (1999). Disaster Debris Management - Planning Tools. Final Reports. Retrieved from

_Planning_Tools/download https://www.researchgate.net/publication/237778695_Disaster_Debris_Management_-

Robin des Bois. (2011a). Des millions de passagers clandestins tournent autour de l'océan pacifique. Retrieved from http://www.robindesbois.org/des-millions-de-passagers-clandestins-tournent-autour-de-locean-pacifique-2/

Robin des Bois. (2011b). Les déchets en Guadeloupe : constats et perspectives. Retrieved from http://www.robindesbois.org/wp-content/uploads/Guadeloupe Robin des Bois HD.pdf

Robin des Bois. (2018). Les déchets de l'ouragan Irma. Retrieved from http://www.robindesbois.org/wpcontent/uploads/IRMA-RobindesBois-Oct2018-MD.pdf

Roper, W. E. (2008). Waste management policy revisions: lessons learned from the Katrina disaster. International journal of environmental technology and management, vol. 8, $n^{\circ}$ 2-3, 275-309. 
SWANA. (2005). Hurricane Katrina disaster debris management: lessons learned from State and Local governments. Briefing Report. Retrieved from https://swana.org/Portals/0/News/2005/HurricaneKatrinaDisasterDebrisManagement Report-12-22-05.pdf

Tansel, B., Whelan, M., \& Barrera, S. (1994). Building performance and structural waste generation by hurricane Andrew. International journal for housing science and its application, vol. 18, 69-77.

Thompson, B. K., Escobedo, F. J., Staudhammer, C. L., Matyas, C. J., \& Qiu, Y. (2011). Modeling hurricane-caused urban forest debris in Houston, Texas. Landscape and Urban Planning, 101(3), 286-297. doi:10.1016/j.landurbplan.2011.02.034

Umpierre, D., \& Margoles, G. (2011). Broward county's wab-based hurricane debris estimation tool (HurDET). 12.

UN Environment, \& OCHA Joint Unit. (2008). Adressing Disaster Waste Management Issues II. Follow up Mission Report. Turks and Caicos Islands. Retrieved from http://www.unocha.org/sites/dms/Documents/2008 December AddressingDisasterWasteManagementIssues2.pdf

UN Environment, \& OCHA Joint Unit. (2017). Management of post-hurricane disaster waste. Retrieved from http://www.eecentre.org/wp-content/uploads/2017/11/Disaster-waste-management-Dominica-Oct-2017-final-draftformatted.pdf

UNDP Barbados, \& OECS. (2007). Post-disaster Early Recovery in a Caribbean Small Island Developing State. The Case of Hurricane Ivan in Grenada (2004): Best Practices and Lessons Learned. Retrieved from http://www.latinamerica.undp.org/content/dam/rblac/docs/Research and Publications/Crisis Prevention and Recovery/UNDP_RBLAC_The CaseofHurricaneIvan.pdf

UNOCHA. (2011). Disaster waste management Guidelines. Retrieved from Genève: https://www.unocha.org/sites/unocha/files/DWMG.pdf

Van der Sommen, F. J., Pearson, D. M., \& Boggs, G. S. (2018). Analysis of the interrelationship between houses, trees and damage in a cyclone affected city: Can landscape design and planning utilising trees minimise cyclone impact? International Journal of Disaster Risk Reduction, 28, 701-710. doi:10.1016/j.ijdrr.2018.01.031

World Bank. (2004). Grenada : Hurricane Ivan preliminary assessment of damages September 17. Retrieved from http://documents.worldbank.org/curated/en/883271468252659671/Grenada-Hurricane-Ivan-preliminary-assessmentof-damages-September-17-2004 